Table of DOE-STD-1027-92

Hazard Category 3 Threshold Quantities

for the ICRP-30 List of 757 Radionuclides

LANL Fact Sheet

Jim Clow

John Elder

George Heindel

William Inkret

Guthrie Miller 


\section{DISCLAIMER}

This report was prepared as an account of work sponsored by an agency of the United States Government. Neither the United States Government nor any agency thereof, nor any of their employees, make any warranty, express or implied, or assumes any legal liability or responsibility for the accuracy, completeness, or usefulness of any information, apparatus, product, or process disclosed, or represents that its use would not infringe privately owned rights. Reference herein to any specific commercial product, process, or service by trade name, trademark, manufacturer, or otherwise does not necessarily constitute or imply its endorsement, recommendation, or favoring by the United States Government or any agency thereof. The views and opinions of authors expressed herein do not necessarily state or reflect those of the United States Government or any agency thereof. 


\section{DISCLAIMER}

Portions of this document may be illegible in electronic image products. Images are produced from the best available original document. 


\title{
TABLE OF DOE-STD-1027-92 HAZARD CATEGORY 3 THRESHOLD QUANTITIES FOR THE ICRP-30 LIST \\ OF 757 RADIONUCLIDES
}

\section{LANL FACT SHEET}

by

Jim Clow, John Elder, George Heindel, William Inkret, and Guthrie Miller

\begin{abstract}
A table of DOE-STD-1027-92 ${ }^{1}$ Hazard Category 3 threshold quantities, in units of curies and grams, is presented for the International Commission on Radiological Protection-30 (ICRP-30) list of 757 radionuclides. ${ }^{2}$ The specific activity $(\mathrm{Ci} / \mathrm{gm})$ used to convert the threshold quantities from curies to grams is also calculated and tabulated. The half-life values used to generate the specific activities are those specified in ICRP-30.
\end{abstract}

Purpose

To ensure more consistency in Los Alamos National Laboratory (LANL) Safety Analysis Reports (SARs) and safety analysis work, Environmental, Safety, and Health Group 3 (ESH-3) will prepare and maintain LANL Fact Sheets for data used in SARs.

Scope

This fact sheet contains a table with the following:

1. Hazard Category 3 Threshold Quantities determined as in DOESTD-1027-92 and expressed in curies

2. Hazard Category 3 Threshold Quantities determined as in DOESTD-1027-92 and expressed in grams

3. Calculated specific activity (SA) used to convert the International Commission on Radiological Protection (ICRP)-30 Radionuclide (column 1) and obtain Category 3 threshold values in curies (column 2)

4. The radiation-dose pathways that determine threshold quantities 
Background

Release Pathways

Calculated Threshold Values
The Hazard Category 3 Threshold Quantity listed in the DOE-STD1027-92 document defines the minimum quantity of a radionuclide that would cause a DOE facility to be classified as a nuclear facility. We determined the Hazard Category 3 Threshold Quantities by multiplying by 20 the Environmental Protection Agency (EPA) threshold quantities from 40 CFR 302.4. ${ }^{3}$ In general, the methodology is the same used to calculate the shorter table of values given in DOE-STD-1027-92 Attachment 1.

LANL ESH-3 will issue Fact Sheet revisions as needed.

DOE-STD-1027-92 Hazard Category 3 threshold quantities were calculated from the smallest of the EPA release values for the four pathways considered by the EPA. The pathways listed in column 5 are water (w), food (f), direct exposure (d), and inhalation (i); the smallest pathway-release value defines the threshold. Two abbreviations indicate both pathways have equal, minimum release values. See reference number 3 for a complete description of the methodology used.

We placed the DOE-STD-1027-92 threshold values in the table if the quantity was within 5\% of our calculated values. ESH-3-calculated thresholds may differ from DOE-STD-1027-92 threshold quantities because the authors chose to carry all calculations to three significant digits instead of two. All of the DOE-Standard-1027-92 Hazard Category 3 threshold quantities expressed in curies agreed with our calculated quantities. However, conversion to grams showed a disagreement in the following six cases:

$\mathrm{Fe}-59$

$\mathrm{Sb}-126$

$\mathrm{Te}-127 \mathrm{~m}$

$\mathrm{Hg}-203$

$\mathrm{Bi}-207$

Cf-252

For $\mathrm{Fe}-59, \mathrm{Sb}-126, \mathrm{Te}-127 \mathrm{~m}$, and $\mathrm{Hg}-203$, the disagreement is a multiple of ten and suggests a typographical error in DOE-STD1027-92. Reference number 4 discusses a possible source of disagreement for $\mathrm{Bi}-207$. We have no explanation for the disagreement for $\mathrm{Cf}-252$. 
Reference numbers in the table refer to Notes, Explanations, and References on page 26.

\section{DOE-STD-1027-92 Hazard Category 3 Threshold Quantities for the ICRP-30 List of 757 Radionuclides}

\begin{tabular}{|c|c|c|c|c|}
\hline $\begin{array}{l}\text { ICRP-30 } \\
\text { Radionuclide }\end{array}$ & $\begin{array}{c}\text { Category } 3 \\
\text { Threshold } \\
\text { (Curies) }\end{array}$ & $\begin{array}{c}\text { Category } 3 \\
\text { Threshold (Grams) }\end{array}$ & $\begin{array}{c}\text { Calculated SA } \\
(\mathrm{Ci} / \mathrm{gm})^{5}\end{array}$ & Pathway \\
\hline$H-3^{(6)}$ & $1.00 \mathrm{E}+03$ & $1.00 \mathrm{E}-01$ & & \\
\hline $\mathrm{Be}-7$ & $1.48 \mathrm{E}+04$ & $4.23 \mathrm{E}-02$ & $3.50 \mathrm{E}+05$ & $d$ \\
\hline Be-10 & $1.04 \mathrm{E}+02$ & $4.65 E+03$ & $2.24 \mathrm{E}-02$ & $\mathrm{i}$ \\
\hline C-11 & $3.60 \mathrm{E}+04$ & $4.29 \mathrm{E}-05$ & $8.39 E+08$ & $d$ \\
\hline C-14 & $4.20 \mathrm{E}+02$ & $9.40 \mathrm{E}+01$ & $4.46 \mathrm{E}+00$ & $\mathrm{i}$ \\
\hline F-18 & $7.20 \mathrm{E}+05$ & 7.57E-03 & $9.52 E+07$ & $\mathrm{i}$ \\
\hline $\mathrm{Na}-22$ & $2.40 \mathrm{E}+02$ & $3.80 \mathrm{E}-02$ & $6.25 E+03$ & $\mathrm{f}$ \\
\hline $\mathrm{Na}-24$ & $3.00 \mathrm{E}+02$ & $3.45 \mathrm{E}-05$ & $8.70 \mathrm{E}+06$ & d \\
\hline $\mathrm{Mg}-28$ & $7.40 \mathrm{E}+02$ & $1.38 \mathrm{E}-04$ & $5.35 E+06$ & d \\
\hline A1-26 & $2.40 \mathrm{E}+02$ & $1.25 E+04$ & $1.92 \mathrm{E}-02$ & $\mathrm{f}$ \\
\hline Si-31 & $3.20 \mathrm{E}+05$ & 8.30E-03 & $3.86 \mathrm{E}+07$ & $\mathbf{i}$ \\
\hline $\mathrm{Si}-32$ & $5.20 \mathrm{E}+01$ & $2.09 E+00$ & $2.48 E+01$ & $\mathrm{i}$ \\
\hline P-32 & $1.20 \mathrm{E}+01$ & $4.20 \mathrm{E}-05$ & $2.86 \mathrm{E}+05$ & f \\
\hline P-33 & $9.40 \mathrm{E}+01$ & $6.00 \mathrm{E}-04$ & $1.56 \mathrm{E}+05$ & $\mathrm{f}$ \\
\hline S-35 & $7.80 \mathrm{E}+01$ & $1.80 \mathrm{E}-03$ & $4.27 \mathrm{E}+04$ & $\mathrm{f}$ \\
\hline C1-36 & $3.40 \mathrm{E}+02$ & $1.00 \mathrm{E}+04$ & $3.30 \mathrm{E}-02$ & $\mathrm{f}$ \\
\hline $\mathrm{C} 1-38$ & $1.38 \mathrm{E}+04$ & $1.04 \mathrm{E}-04$ & $1.33 E+08$ & d \\
\hline C1-39 & $9.00 E+03$ & $1.06 \mathrm{E}-04$ & $8.52 E+07$ & d \\
\hline Ar-39 & $4.00 \mathrm{E}+04$ & $1.17 \mathrm{E}+03$ & $3.41 \mathrm{E}+01$ & d \\
\hline $\mathrm{Ar}-41$ & $6.00 \mathrm{E}+02$ & $1.43 \mathrm{E}-05$ & $4.18 \mathrm{E}+07$ & d \\
\hline K-40 & $1.70 \mathrm{E}+02$ & $2.40 E+07$ & $6.99 \mathrm{E}-06$ & $\mathrm{f}$ \\
\hline $\mathrm{K}-42$ & $4.60 E+03$ & 7.62E-04 & $6.04 E+06$ & d \\
\hline $\mathrm{K}-43$ & $1.16 \mathrm{E}+03$ & $3.60 \mathrm{E}-04$ & $3.23 E+06$ & d \\
\hline K-44 & $1.34 \mathrm{E}+04$ & $6.94 \mathrm{E}-05$ & $1.93 E+08$ & d \\
\hline $\mathrm{K}-45$ & $2.40 \mathrm{E}+04$ & $1.15 \mathrm{E}-04$ & $2.09 E+08$ & d \\
\hline $\mathrm{Ca}-41$ & $1.60 \mathrm{E}+03$ & $2.57 E+04$ & $6.23 E-02$ & f \\
\hline $\mathrm{Ca}-45$ & $1.10 \mathrm{E}+03$ & $6.20 \mathrm{E}-02$ & $1.78 \mathrm{E}+04$ & $\mathrm{f}$ \\
\hline $\mathrm{Ca}-47$ & $7.00 \mathrm{E}+02$ & $1.10 \mathrm{E}-03$ & $6.13 E+05$ & d \\
\hline Sc-43 & $3.80 \mathrm{E}+04$ & $2.03 E-03$ & $1.87 \mathrm{E}+07$ & d \\
\hline Sc- $-44 \mathrm{~m}$ & $1.90 \mathrm{E}+03$ & $1.56 \mathrm{E}-03$ & $1.22 E+06$ & $\mathrm{f}$ \\
\hline Sc-44 & $2.60 \mathrm{E}+03$ & $1.43 \mathrm{E}-04$ & $1.81 \mathrm{E}+07$ & d \\
\hline
\end{tabular}




\begin{tabular}{|c|c|c|c|c|}
\hline $\begin{array}{c}\text { ICRP-30 } \\
\text { Radionuclide }\end{array}$ & $\begin{array}{l}\text { Category } 3 \\
\text { Threshold } \\
\text { (Curies) }\end{array}$ & $\begin{array}{c}\text { Category } 3 \\
\text { Threshold (Grams) }\end{array}$ & $\begin{array}{l}\text { Calculated SA } \\
(\mathrm{Ci} / \mathrm{gm})^{5}\end{array}$ & Pathway \\
\hline Sc- 46 & $3.60 \mathrm{E}+02$ & $1.10 \mathrm{E}-02$ & $3.39 \mathrm{E}+04$ & d \\
\hline Sc- 47 & $5.80 \mathrm{E}+03$ & $7.00 \mathrm{E}-03$ & $8.29 E+05$ & f \\
\hline Sc-48 & $2.60 \mathrm{E}+02$ & $1.74 \mathrm{E}-04$ & $1.49 E+06$ & d \\
\hline Sc-49 & $5.20 \mathrm{E}+05$ & $7.78 \mathrm{E}-03$ & $6.68 \mathrm{E}+07$ & $\mathrm{i}$ \\
\hline Ti-44 & $6.20 \mathrm{E}+01$ & $3.60 \mathrm{E}-01$ & $1.72 \mathrm{E}+02$ & $\mathbf{i}$ \\
\hline Ti-45 & $3.20 \mathrm{E}+05$ & $1.41 \mathrm{E}-02$ & $2.26 \mathrm{E}+07$ & i \\
\hline$V-47$ & $8.20 \mathrm{E}+05$ & $6.68 \mathrm{E}-03$ & $1.23 \mathrm{E}+08$ & $\mathrm{i}$ \\
\hline V-48 & $6.40 \mathrm{E}+02$ & $3.80 \mathrm{E}-03$ & $1.68 E+05$ & f \\
\hline V-49 & $4.40 \mathrm{E}+04$ & $5.45 \mathrm{E}+00$ & $8.08 E+03$ & f \\
\hline $\mathrm{Cr}-48$ & $2.60 \mathrm{E}+03$ & $9.14 \mathrm{E}-04$ & $2.84 E+06$ & d \\
\hline $\mathrm{Cr}-49$ & $1.96 \mathrm{E}+05$ & $2.15 \mathrm{E}-03$ & $9.12 \mathrm{E}+07$ & d \\
\hline $\mathrm{Cr}-51$ & $2.20 \mathrm{E}+04$ & 2.40E-01 & $9.24 \mathrm{E}+04$ & d \\
\hline $\mathrm{Mn}-51$ & $5.20 \mathrm{E}+05$ & $6.52 \mathrm{E}-03$ & $7.98 \mathrm{E}+07$ & $\mathbf{i}$ \\
\hline$M n-52 m$ & $2.40 \mathrm{E}+04$ & $1.40 \mathrm{E}-04$ & $1.71 E+08$ & d \\
\hline $\mathrm{Mn}-52$ & $3.40 \mathrm{E}+02$ & $7.60 \mathrm{E}-04$ & $4.49 E+05$ & d \\
\hline Mn-53 & $3.00 \mathrm{E}+04$ & $1.64 E+07$ & $1.82 \mathrm{E}-03$ & f \\
\hline Mn-54 & $8.80 \mathrm{E}+02$ & $1.14 \mathrm{E}-01$ & $7.74 \mathrm{E}+03$ & d \\
\hline $\mathrm{Mn}-56$ & $2.80 \mathrm{E}+03$ & $1.29 \mathrm{E}-04$ & $2.17 \mathrm{E}+07$ & d \\
\hline $\mathrm{Fe}-52$ & $1.00 \mathrm{E}+04$ & $1.37 \mathrm{E}-03$ & $7.28 \mathrm{E}+06$ & d \\
\hline $\mathrm{Fe}-55$ & $5.40 \mathrm{E}+03$ & $2.20 \mathrm{E}+00$ & $2.41 E+03$ & $\mathrm{f}$ \\
\hline $\mathrm{Fe}-59$ & $6.00 \mathrm{E}+02$ & $1.21 \mathrm{E}-02$ & $4.97 E+04$ & $\mathrm{f}$ \\
\hline $\mathrm{Fe}-60$ & $1.78 \mathrm{E}+01$ & $2.99 \mathrm{E}+02$ & $5.96 \mathrm{E}-02$ & f \\
\hline Co-55 & $9.80 \mathrm{E}+02$ & $3.02 \mathrm{E}-04$ & $3.25 E+06$ & d \\
\hline $\mathrm{Co}-56$ & $2.20 \mathrm{E}+02$ & $7.43 E-03$ & $2.96 \mathrm{E}+04$ & $\mathrm{~d}$ \\
\hline $\mathrm{Co}-57$ & $6.00 \mathrm{E}+03$ & $7.09 \mathrm{E}-01$ & $8.46 E+03$ & d \\
\hline $\mathrm{Co}-58 \mathrm{~m}$ & $6.20 E+06$ & $1.05 E+00$ & $5.91 \mathrm{E}+06$ & i \\
\hline $\mathrm{Co}-58$ & $9.00 \mathrm{E}+02$ & $2.83 \mathrm{E}-02$ & $3.18 \mathrm{E}+04$ & $\mathrm{~d}$ \\
\hline $\mathrm{Co}-60 \mathrm{~m}$ & $5.80 \mathrm{E}+07$ & $1.94 \mathrm{E}-01$ & $2.99 \mathrm{E}+08$ & d \\
\hline $\mathrm{Co}-60$ & $2.80 \mathrm{E}+02$ & $2.50 \mathrm{E}-01$ & $1.13 E+03$ & $d$ \\
\hline $\mathrm{Co}-61$ & $8.00 \mathrm{E}+04$ & $2.57 \mathrm{E}-03$ & $3.11 \mathrm{E}+07$ & $d$ \\
\hline $\mathrm{Co}-62 \mathrm{~m}$ & $2.00 \mathrm{E}+04$ & 9.17E-05 & $2.18 E+08$ & d \\
\hline $\mathrm{Ni}-56$ & $4.60 \mathrm{E}+02$ & $1.20 \mathrm{E}-03$ & $3.82 E+05$ & $\mathrm{~d}$ \\
\hline $\mathrm{Ni}-57$ & $6.00 \mathrm{E}+02$ & $3.94 \mathrm{E}-04$ & $1.52 \mathrm{E}+06$ & $\mathrm{~d}$ \\
\hline $\mathrm{Ni}-59$ & $1.18 \mathrm{E}+04$ & $1.46 \mathrm{E}+05$ & $8.08 \mathrm{E}-02$ & $\mathrm{f}$ \\
\hline
\end{tabular}




\begin{tabular}{|c|c|c|c|c|}
\hline $\begin{array}{l}\text { ICRP-30 } \\
\text { Radionuclide }\end{array}$ & $\begin{array}{c}\text { Category } 3 \\
\text { Threshold } \\
\text { (Curies) } \\
\end{array}$ & $\begin{array}{c}\text { Category } 3 \\
\text { Threshold (Grams) }\end{array}$ & $\begin{array}{c}\text { Calculated SA } \\
(\mathrm{Ci} / \mathrm{gm})^{5}\end{array}$ & Pathway \\
\hline $\mathrm{Ni}-63$ & $5.40 \mathrm{E}+03$ & $9.50 \mathrm{E}+01$ & $5.91 \mathrm{E}+01$ & $\mathrm{f}$ \\
\hline $\mathrm{Ni}-65$ & $9.00 \mathrm{E}+03$ & 4.70E-04 & $1.91 \mathrm{E}+07$ & d \\
\hline $\mathrm{Ni}-66$ & $1.62 \mathrm{E}+03$ & $1.86 \mathrm{E}-03$ & $8.70 E+05$ & f \\
\hline $\mathrm{Cu}-60$ & $1.98 \mathrm{E}+04$ & $1.47 \mathrm{E}-04$ & $1.35 \mathrm{E}+08$ & d \\
\hline $\mathrm{Cu}-61$ & $1.64 E+04$ & $1.09 E-03$ & $1.51 \mathrm{E}+07$ & d \\
\hline $\mathrm{Cu}-64$ & $1.54 \mathrm{E}+05$ & $3.99 \mathrm{E}-02$ & $3.86 \mathrm{E}+06$ & $\mathrm{f}$ \\
\hline $\mathrm{Cu}-67$ & $7.20 \mathrm{E}+03$ & $9.52 E-03$ & $7.56 \mathrm{E}+05$ & d \\
\hline $\mathrm{Zn}-62$ & $5.20 \mathrm{E}+03$ & $9.53 \mathrm{E}-04$ & $5.46 \mathrm{E}+06$ & d \\
\hline $\mathrm{Zn}-63$ & $1.40 \mathrm{E}+05$ & $1.79 \mathrm{E}-03$ & $7.83 E+07$ & d \\
\hline $\mathrm{Zn}-65$ & $2.40 \mathrm{E}+02$ & $2.90 \mathrm{E}-02$ & $8.24 \mathrm{E}+03$ & $\mathrm{f}$ \\
\hline $\mathrm{Zn}-69 \mathrm{~m}$ & $3.00 \mathrm{E}+03$ & $9.09 \mathrm{E}-04$ & $3.30 \mathrm{E}+06$ & d \\
\hline $\mathrm{Zn}-69$ & $1.04 \mathrm{E}+06$ & $2.17 \mathrm{E}-02$ & $4.78 \mathrm{E}+07$ & $\mathrm{i}$ \\
\hline $\mathrm{Zn}-71 \mathrm{~m}$ & $2.20 \mathrm{E}+03$ & $1.94 \mathrm{E}-04$ & $1.13 E+07$ & d \\
\hline $\mathrm{Zn}-72$ & $4.60 \mathrm{E}+03$ & $4.91 \mathrm{E}-03$ & $9.36 \mathrm{E}+05$ & $\mathrm{f}$ \\
\hline $\mathrm{Ga}-65$ & $1.14 E+05$ & $5.99 \mathrm{E}-04$ & $1.90 \mathrm{E}+08$ & d \\
\hline $\mathrm{Ga}-66$ & $1.10 \mathrm{E}+03$ & $2.18 \mathrm{E}-04$ & $5.05 E+06$ & d \\
\hline $\mathrm{Ga}-67$ & $4.80 \mathrm{E}+03$ & 8.03E-03 & $5.98 E+05$ & d \\
\hline Ga-68 & $3.20 \mathrm{E}+05$ & $7.87 \mathrm{E}-03$ & $4.07 \mathrm{E}+07$ & d \\
\hline $\mathrm{Ga}-70$ & $2.00 \mathrm{E}+06$ & $1.57 \mathrm{E}-02$ & $1.27 \mathrm{E}+08$ & $\mathrm{i}$ \\
\hline $\mathrm{Ga}-72$ & $7.20 \mathrm{E}+02$ & $2.33 E-04$ & $3.09 E+06$ & d \\
\hline $\mathrm{Ga}-73$ & $1.44 \mathrm{E}+04$ & $1.65 \mathrm{E}-03$ & $8.75 E+06$ & d \\
\hline Ge-66 & $1.68 \mathrm{E}+04$ & 8.03E-04 & $2.09 E+07$ & d \\
\hline Ge-67 & $1.08 \mathrm{E}+05$ & $7.20 \mathrm{E}-04$ & $1.50 \mathrm{E}+08$ & d \\
\hline $\mathrm{Ge}-68$ & $1.00 \mathrm{E}+03$ & $1.50 \mathrm{E}-01$ & $6.67 E+03$ & $\mathrm{i}$ \\
\hline Ge-69 & $1.02 \mathrm{E}+03$ & 8.77E-04 & $1.16 E+06$ & d \\
\hline $\mathrm{Ge}-71$ & $4.20 \mathrm{E}+05$ & $2.69 \mathrm{E}+00$ & $1.56 \mathrm{E}+05$ & i \\
\hline Ge-75 & $3.00 \mathrm{E}+05$ & $9.90 \mathrm{E}-03$ & $3.03 E+07$ & d \\
\hline $\mathrm{Ge}-77$ & $1.92 E+03$ & 5.33E-04 & $3.60 \mathrm{E}+06$ & d \\
\hline Ge-78 & $3.00 E+04$ & $1.08 \mathrm{E}-03$ & $2.77 \mathrm{E}+07$ & d \\
\hline As-69 & $1.04 \mathrm{E}+06$ & $5.80 \mathrm{E}-03$ & $1.79 E+08$ & $\mathrm{i}$ \\
\hline As-70 & $5.80 \mathrm{E}+03$ & $1.14 \mathrm{E}-04$ & $5.11 E+07$ & d \\
\hline As-71 & $3.80 \mathrm{E}+03$ & $5.58 \mathrm{E}-03$ & $6.81 E+05$ & d \\
\hline As-72 & $1.12 E+03$ & $6.69 \mathrm{E}-04$ & $1.67 \mathrm{E}+06$ & d \\
\hline As-73 & $5.40 \mathrm{E}+03$ & $2.42 \mathrm{E}-01$ & $2.23 E+04$ & f \\
\hline
\end{tabular}


Table-Continued

\begin{tabular}{lcccc}
\hline \hline $\begin{array}{c}\text { ICRP-30 } \\
\text { Radionuclide }\end{array}$ & $\begin{array}{c}\text { Category } 3 \\
\text { Threshold } \\
\text { (Curies) }\end{array}$ & $\begin{array}{c}\text { Category } 3 \\
\text { Threshold (Grams) }\end{array}$ & $\begin{array}{c}\text { Calculated SA } \\
(\mathrm{Ci} / \mathrm{gm})^{5}\end{array}$ & Pathway \\
\hline & & & & \\
As-74 & $1.02 \mathrm{E}+03$ & $1.03 \mathrm{E}-02$ & $9.94 \mathrm{E}+04$ & $\mathrm{f}$ \\
As-76 & $2.60 \mathrm{E}+03$ & $1.66 \mathrm{E}-03$ & $1.57 \mathrm{E}+06$ & $\mathrm{~d}$ \\
As-77 & $2.20 \mathrm{E}+04$ & $2.10 \mathrm{E}-02$ & $1.05 \mathrm{E}+06$ & $\mathrm{f}$ \\
$\mathrm{As}-78$ & $7.80 \mathrm{E}+03$ & $2.93 \mathrm{E}-04$ & $2.66 \mathrm{E}+07$ & $\mathrm{~d}$ \\
$\mathrm{Se}-70$ & $9.20 \mathrm{E}+04$ & $1.40 \mathrm{E}-03$ & $6.55 \mathrm{E}+07$ & $\mathrm{~d}$ \\
$\mathrm{Se}-73 \mathrm{~m}$ & $3.40 \mathrm{E}+03$ & $5.15 \mathrm{E}-05$ & $6.61 \mathrm{E}+07$ & $\mathrm{~d}$ \\
$\mathrm{Se}-73$ & $1.90 \mathrm{E}+03$ & $3.16 \mathrm{E}-04$ & $6.01 \mathrm{E}+06$ & $\mathrm{~d}$ \\
$\mathrm{Se}-75$ & $3.20 \mathrm{E}+02$ & $2.20 \mathrm{E}-02$ & $1.45 \mathrm{E}+04$ & $\mathrm{f}$ \\
$\mathrm{Se}-79$ & $3.60 \mathrm{E}+02$ & $5.17 \mathrm{E}+03$ & $6.97 \mathrm{E}-02$ & $\mathrm{f}$ \\
$\mathrm{Se}-81 \mathrm{~m}$ & $7.20 \mathrm{E}+05$ & $1.78 \mathrm{E}-02$ & $4.05 \mathrm{E}+07$ & $\mathrm{i}$ \\
$\mathrm{Se}-81$ & $2.00 \mathrm{E}+06$ & $1.59 \mathrm{E}-02$ & $1.25 \mathrm{E}+08$ & $\mathrm{i}$ \\
$\mathrm{Se}-83$ & $2.00 \mathrm{E}+04$ & $1.99 \mathrm{E}-04$ & $1.01 \mathrm{E}+08$ & $\mathrm{~d}$ \\
$\mathrm{Br}-74 \mathrm{~m}$ & $6.40 \mathrm{E}+03$ & $1.05 \mathrm{E}-04$ & $6.12 \mathrm{E}+07$ & $\mathrm{~d}$ \\
$\mathrm{Br}-74$ & $1.92 \mathrm{E}+04$ & $1.91 \mathrm{E}-04$ & $1.00 \mathrm{E}+08$ & $\mathrm{~d}$ \\
$\mathrm{Br}-75$ & $6.60 \mathrm{E}+03$ & $2.58 \mathrm{E}-04$ & $2.56 \mathrm{E}+07$ & $\mathrm{~d}$ \\
$\mathrm{Br}-76$ & $5.60 \mathrm{E}+02$ & $2.20 \mathrm{E}-04$ & $2.55 \mathrm{E}+06$ & $\mathrm{~d}$ \\
$\mathrm{Br}-77$ & $3.00 \mathrm{E}+03$ & $4.13 \mathrm{E}-03$ & $7.27 \mathrm{E}+05$ & $\mathrm{~d}$ \\
$\mathrm{Br}-80 \mathrm{~m}$ & $1.04 \mathrm{E}+05$ & $1.17 \mathrm{E}-02$ & $8.87 \mathrm{E}+06$ & $\mathrm{i}$ \\
$\mathrm{Br}-80$ & $8.00 \mathrm{E}+05$ & $5.92 \mathrm{E}-03$ & $1.35 \mathrm{E}+08$ & $\mathrm{~d}$ \\
$\mathrm{Br}-82$ & $3.40 \mathrm{E}+02$ & $3.14 \mathrm{E}-04$ & $1.08 \mathrm{E}+06$ & $\mathrm{~d}$ \\
$\mathrm{Br}-83$ & $6.20 \mathrm{E}+05$ & $3.92 \mathrm{E}-02$ & $1.58 \mathrm{E}+07$ & $\mathrm{i}$ \\
$\mathrm{Br}-84$ & $1.38 \mathrm{E}+04$ & $1.96 \mathrm{E}-04$ & $7.04 \mathrm{E}+07$ & $\mathrm{~d}$ \\
$\mathrm{Kr}-74$ & $6.00 \mathrm{E}+02$ & $2.71 \mathrm{E}-06$ & $2.21 \mathrm{E}+08$ & $\mathrm{~d}$ \\
$\mathrm{Kr}-76$ & $1.80 \mathrm{E}+03$ & $6.46 \mathrm{E}-04$ & $2.79 \mathrm{E}+06$ & $\mathrm{~d}$ \\
$\mathrm{Kr}-77$ & $8.00 \mathrm{E}+02$ & $2.45 \mathrm{E}-05$ & $3.27 \mathrm{E}+07$ & $\mathrm{~d}$ \\
$\mathrm{Kr}-79$ & $4.00 \mathrm{E}+03$ & $3.53 \mathrm{E}-03$ & $1.13 \mathrm{E}+06$ & $\mathrm{~d}$ \\
$\mathrm{Kr}-81$ & $1.40 \mathrm{E}+05$ & $6.66 \mathrm{E}+06$ & $2.10 \mathrm{E}-02$ & $\mathrm{~d}$ \\
$\mathrm{Kr}-83 \mathrm{~m}$ & $2.00 \mathrm{E}+06$ & $9.69 \mathrm{E}-02$ & $2.06 \mathrm{E}+07$ & $\mathrm{~d}$ \\
$\mathrm{Kr}-85 \mathrm{~m}$ & $4.00 \mathrm{E}+03$ & $4.86 \mathrm{E}-04$ & $8.23 \mathrm{E}+06$ & $\mathrm{~d}$ \\
$\mathrm{Kr}-85$ & $2.00 \mathrm{E}+04$ & $5.10 \mathrm{E}+01$ & $3.93 \mathrm{E}+02$ & $\mathrm{~d}$ \\
$\mathrm{Kr}-87$ & $1.00 \mathrm{E}+03$ & $3.53 \mathrm{E}-05$ & $2.83 \mathrm{E}+07$ & $\mathrm{~d}$ \\
$\mathrm{Kr}-88$ & $4.00 \mathrm{E}+02$ & $3.19 \mathrm{E}-05$ & $1.25 \mathrm{E}+07$ & $\mathrm{~d}$ \\
$\mathrm{Rb}-79$ & $2.40 \mathrm{E}+04$ & $2.31 \mathrm{E}-04$ & $1.04 \mathrm{E}+08$ & $\mathrm{~d}$ \\
$\mathrm{Rb}-81 \mathrm{~m}$ & $9.60 \mathrm{E}+04$ & $1.32 \mathrm{E}-03$ & $7.26 \mathrm{E}+07$ & Continued on next page
\end{tabular}


Table-Continued

\begin{tabular}{|c|c|c|c|c|}
\hline $\begin{array}{l}\text { ICRP-30 } \\
\text { Radionuclide }\end{array}$ & $\begin{array}{c}\text { Category } 3 \\
\text { Threshold } \\
\text { (Curies) }\end{array}$ & $\begin{array}{c}\text { Category } 3 \\
\text { Threshold (Grams) }\end{array}$ & $\begin{array}{l}\text { Calculated SA } \\
(\mathrm{Ci} / \mathrm{gm})^{5}\end{array}$ & Pathway \\
\hline $\mathrm{Rb}-81$ & $5.80 \mathrm{E}+03$ & $6.86 \mathrm{E}-04$ & $8.45 E+06$ & d \\
\hline $\mathrm{Rb}-82 \mathrm{~m}$ & $7.80 \mathrm{E}+02$ & $1.27 \mathrm{E}-04$ & $6.17 \mathrm{E}+06$ & $d$ \\
\hline $\mathrm{Rb}-83$ & $4.00 \mathrm{E}+02$ & $2.19 \mathrm{E}-02$ & $1.83 \mathrm{E}+04$ & f \\
\hline $\mathrm{Rb}-84$ & $4.00 \mathrm{E}+02$ & $8.43 \mathrm{E}-03$ & $4.74 E+04$ & f \\
\hline $\mathrm{Rb}-86$ & $5.00 \mathrm{E}+02$ & $6.14 \mathrm{E}-03$ & $8.14 \mathrm{E}+04$ & f \\
\hline $\mathrm{Rb}-87$ & $6.00 \mathrm{E}+02$ & $6.86 E+09$ & $8.75 \mathrm{E}-08$ & f \\
\hline $\mathrm{Rb}-88$ & $5.80 \mathrm{E}+04$ & 4.83E-04 & $1.20 \mathrm{E}+08$ & d \\
\hline $\mathrm{Rb}-89$ & $2.60 \mathrm{E}+04$ & $1.87 \mathrm{E}-04$ & $1.39 \mathrm{E}+08$ & d \\
\hline Sr -80 & $8.20 \mathrm{E}+03$ & $3.49 \mathrm{E}-04$ & $2.35 \mathrm{E}+07$ & d \\
\hline $\mathrm{Sr}-81$ & $2.60 \mathrm{E}+04$ & $2.86 \mathrm{E}-04$ & $9.10 \mathrm{E}+07$ & d \\
\hline $\mathrm{Sr}-83$ & $2.60 \mathrm{E}+03$ & $2.23 \mathrm{E}-03$ & $1.17 \mathrm{E}+06$ & d \\
\hline Sr-85m & $5.40 \mathrm{E}+04$ & $1.70 \mathrm{E}-03$ & $3.18 \mathrm{E}+07$ & d \\
\hline Sr-85 & $1.44 \mathrm{E}+03$ & $6.08 \mathrm{E}-02$ & $2.37 E+04$ & d \\
\hline Sr-87m & $1.36 \mathrm{E}+04$ & $1.06 \mathrm{E}-03$ & $1.28 \mathrm{E}+07$ & d \\
\hline Sr-89 & $3.40 \mathrm{E}+02$ & $1.20 \mathrm{E}-02$ & $2.91 \mathrm{E}+04$ & f \\
\hline Sr -90 & $1.60 \mathrm{E}+01$ & $1.20 \mathrm{E}-01$ & $1.36 \mathrm{E}+02$ & $\mathrm{f}$ \\
\hline Sr-91 & $1.58 \mathrm{E}+03$ & $4.36 \mathrm{E}-04$ & $3.63 E+06$ & d \\
\hline Sr-92 & $3.40 \mathrm{E}+03$ & $2.70 \mathrm{E}-04$ & $1.26 E+07$ & d \\
\hline$Y-86 m$ & $7.80 E+04$ & $1.71 \mathrm{E}-03$ & $4.56 \mathrm{E}+07$ & d \\
\hline Y-86 & $4.60 \mathrm{E}+02$ & $1.86 \mathrm{E}-04$ & $2.47 E+06$ & d \\
\hline$Y-87$ & $1.00 \mathrm{E}+03$ & $2.23 \mathrm{E}-03$ & $4.49 E+05$ & d \\
\hline Y-88 & $2.80 \mathrm{E}+02$ & $2.01 \mathrm{E}-02$ & $1.39 E+04$ & d \\
\hline$Y-90 m$ & $6.00 \mathrm{E}+03$ & $5.50 \mathrm{E}-04$ & $1.09 \mathrm{E}+07$ & d \\
\hline$Y-90$ & $1.42 E+03$ & $2.61 \mathrm{E}-03$ & $5.44 \mathrm{E}+05$ & $\mathrm{f}$ \\
\hline $\mathrm{Y}-91 \mathrm{~m}$ & $2.80 E+04$ & $6.74 \mathrm{E}-04$ & $4.16 \mathrm{E}+07$ & d \\
\hline Y-91 & $3.60 \mathrm{E}+02$ & $1.50 \mathrm{E}-02$ & $2.45 E+04$ & $\mathrm{f}$ \\
\hline Y-92 & $1.40 \mathrm{E}+04$ & $1.45 \mathrm{E}-03$ & $9.63 E+06$ & d \\
\hline Y-93 & $1.76 E+04$ & $5.27 \mathrm{E}-03$ & $3.34 E+06$ & d \\
\hline Y-94 & $2.80 \mathrm{E}+04$ & 2.67E-04 & $1.05 E+08$ & d \\
\hline Y-95 & $6.20 \mathrm{E}+04$ & 3.35E-04 & $1.85 E+08$ & d \\
\hline $\mathrm{Zr}-86$ & $4.40 E+03$ & $1.99 \mathrm{E}-03$ & $2.21 E+06$ & d \\
\hline $\mathrm{Zr}-88$ & $1.92 \mathrm{E}+03$ & $1.08 \mathrm{E}-01$ & $1.78 E+04$ & d \\
\hline $\mathrm{Zr}-89$ & $3.20 \mathrm{E}+03$ & 7.13E-03 & $4.49 E+05$ & $\mathrm{~d}$ \\
\hline Zr-93 & $6.20 \mathrm{E}+01$ & $2.50 \mathrm{E}+04$ & $2.51 \mathrm{E}-03$ & $\mathrm{i}$ \\
\hline
\end{tabular}

Continued on next page 
Table-Continued

\begin{tabular}{|c|c|c|c|c|}
\hline $\begin{array}{l}\text { ICRP-30 } \\
\text { Radionuclide }\end{array}$ & $\begin{array}{c}\text { Category } 3 \\
\text { Threshold } \\
\text { (Curies) }\end{array}$ & $\begin{array}{c}\text { Category } 3 \\
\text { Threshold (Grams) }\end{array}$ & $\begin{array}{l}\text { Calculated SA } \\
(\mathrm{Ci} / \mathrm{gm})^{5}\end{array}$ & Pathway \\
\hline $\mathrm{Zr}-95$ & $7.00 \mathrm{E}+02$ & $3.30 \mathrm{E}-02$ & $2.15 E+04$ & f \\
\hline Zr-97 & $1.38 \mathrm{E}+03$ & $7.22 \mathrm{E}-04$ & $1.91 E+06$ & d \\
\hline $\mathrm{Nb}-88$ & $1.58 \mathrm{E}+04$ & $1.06 \mathrm{E}-04$ & $1.49 E+08$ & d \\
\hline $\begin{array}{l}\mathrm{Nb}-89 \\
(66 \mathrm{~min})\end{array}$ & $6.00 \mathrm{E}+03$ & $1.87 \mathrm{E}-04$ & $3.20 \mathrm{E}+07$ & $d$ \\
\hline $\begin{array}{l}\mathrm{Nb}-89 \\
(122 \mathrm{~min})\end{array}$ & $1.62 \mathrm{E}+04$ & 9.35E-04 & $1.73 E+07$ & $d$ \\
\hline $\mathrm{Nb}-90$ & $3.00 \mathrm{E}+02$ & $1.26 \mathrm{E}-04$ & $2.39 \mathrm{E}+06$ & d \\
\hline $\mathrm{Nb}-93 \mathrm{~m}$ & $2.00 E+03$ & $7.07 \mathrm{E}+00$ & $2.83 E+02$ & $\mathbf{i}$ \\
\hline $\mathrm{Nb}-94$ & $2.00 \mathrm{E}+02$ & $1.10 \mathrm{E}+03$ & $1.87 \mathrm{E}-01$ & i \\
\hline $\mathrm{Nb}-95 \mathrm{~m}$ & $5.60 \mathrm{E}+03$ & $1.47 \mathrm{E}-02$ & $3.81 E+05$ & f \\
\hline $\mathrm{Nb}-95$ & $9.60 \mathrm{E}+02$ & $2.45 \mathrm{E}-02$ & $3.91 \mathrm{E}+04$ & d \\
\hline $\mathrm{Nb}-96$ & $4.40 \mathrm{E}+02$ & 3.15E-04 & $1.40 \mathrm{E}+06$ & d \\
\hline $\mathrm{Nb}-97$ & $1.48 \mathrm{E}+04$ & $5.50 \mathrm{E}-04$ & $2.69 E+07$ & $d$ \\
\hline $\mathrm{Nb}-98$ & $5.20 \mathrm{E}+05$ & $1.40 \mathrm{E}-05$ & $3.73 E+10$ & i \\
\hline Mo-90 & $2.80 \mathrm{E}+03$ & $4.56 \mathrm{E}-04$ & $6.14 E+06$ & d \\
\hline Mo-93m & $8.40 \mathrm{E}+02$ & $1.71 \mathrm{E}-04$ & $4.92 E+06$ & $d$ \\
\hline Mo-93 & $2.00 \mathrm{E}+03$ & $1.82 \mathrm{E}+03$ & $1.10 \mathrm{E}+00$ & $\mathrm{i}$ \\
\hline Mo-99 & $3.40 \mathrm{E}+03$ & $7.10 \mathrm{E}-03$ & $4.80 \mathrm{E}+05$ & $d, f$ \\
\hline Mo-101 & $9.00 \mathrm{E}+04$ & $7.07 \mathrm{E}-04$ & $1.27 \mathrm{E}+08$ & $\mathrm{~d}$ \\
\hline Tc-93m & $2.40 \mathrm{E}+04$ & $5.16 \mathrm{E}-04$ & $4.65 \mathrm{E}+07$ & d \\
\hline Tc-93 & $2.80 \mathrm{E}+03$ & $2.28 \mathrm{E}-04$ & $1.23 E+07$ & d \\
\hline Tc-94m & $7.40 \mathrm{E}+03$ & $1.92 \mathrm{E}-04$ & $3.85 E+07$ & d \\
\hline $\mathrm{Tc}-94$ & $9.60 \mathrm{E}+02$ & $1.41 \mathrm{E}-04$ & $6.83 E+06$ & d \\
\hline Tc-96m & $9.40 \mathrm{E}+05$ & $2.47 \mathrm{E}-02$ & $3.80 E+07$ & d \\
\hline Tc-96 & $3.20 \mathrm{E}+02$ & $1.01 \mathrm{E}-03$ & $3.18 E+05$ & d \\
\hline Tc-97m & $2.60 \mathrm{E}+03$ & $1.68 \mathrm{E}-01$ & $1.55 E+04$ & f \\
\hline Tc-97 & $1.78 \mathrm{E}+04$ & $1.26 \mathrm{E}+07$ & $1.42 \mathrm{E}-03$ & f \\
\hline Tc-98 & $4.40 \mathrm{E}+02$ & $5.06 E+05$ & $8.69 \mathrm{E}-04$ & f \\
\hline Tc-99m & $1.70 \mathrm{E}+04$ & $3.23 E-03$ & $5.26 \mathrm{E}+06$ & $d$ \\
\hline Tc-99 & $1.70 \mathrm{E}+03$ & $1.00 \mathrm{E}+05$ & $1.70 \mathrm{E}-02$ & $\mathrm{f}$ \\
\hline Tc-101 & $1.62 E+05$ & $1.24 \mathrm{E}-03$ & $1.31 E+08$ & d \\
\hline Tc-104 & $2.60 \mathrm{E}+04$ & $2.62 \mathrm{E}-04$ & $9.93 E+07$ & d \\
\hline Ru-94 & $2.80 \mathrm{E}+04$ & 7.25E-04 & $3.86 E+07$ & d \\
\hline
\end{tabular}

Continued on next page 
Table-Continued

\begin{tabular}{|c|c|c|c|c|}
\hline $\begin{array}{c}\text { ICRP-30 } \\
\text { Radionuclide }\end{array}$ & $\begin{array}{c}\text { Category } 3 \\
\text { Threshold } \\
\text { (Curies) }\end{array}$ & $\begin{array}{c}\text { Category } 3 \\
\text { Threshold (Grams) }\end{array}$ & $\begin{array}{l}\text { Calculated SA } \\
(\mathrm{Ci} / \mathrm{gm})^{5}\end{array}$ & Pathway \\
\hline Ru-97 & $2.40 \mathrm{E}+03$ & $5.17 \mathrm{E}-03$ & $4.64 E+05$ & d \\
\hline Ru-103 & $1.56 \mathrm{E}+03$ & 4.83E-02 & $3.23 E+04$ & $\mathrm{~d}, \mathrm{f}$ \\
\hline $\mathrm{Ru}-105$ & $4.00 \mathrm{E}+03$ & 5.95E-04 & $6.72 E+06$ & d \\
\hline Ru-106 & $1.00 \mathrm{E}+02$ & $3.00 \mathrm{E}-02$ & $3.35 E+03$ & i \\
\hline $\mathrm{Rh}-99 \mathrm{~m}$ & $4.60 E+03$ & $6.83 \mathrm{E}-04$ & $6.74 \mathrm{E}+06$ & d \\
\hline$R h-99$ & $1.74 E+03$ & $2.11 \mathrm{E}-02$ & $8.24 \mathrm{E}+04$ & d \\
\hline $\mathrm{Rh}-100$ & $4.20 \mathrm{E}+02$ & $2.79 \mathrm{E}-04$ & $1.51 E+06$ & d \\
\hline $\mathrm{Rh}-101 \mathrm{~m}$ & $2.80 \mathrm{E}+03$ & $9.40 \mathrm{E}-03$ & $2.98 E+05$ & d \\
\hline Rh-101 & $1.20 E+03$ & $1.08 \mathrm{E}+00$ & $1.11 \mathrm{E}+03$ & f \\
\hline Rh-102m & $6.20 E+02$ & $1.00 \mathrm{E}-01$ & $6.18 E+03$ & f \\
\hline Rh-102 & $2.80 E+02$ & $2.32 \mathrm{E}-01$ & $1.21 E+03$ & d \\
\hline $\mathrm{Rh}-103 \mathrm{~m}$ & $1.04 \mathrm{E}+07$ & $3.20 \mathrm{E}-01$ & $3.25 E+07$ & $\mathrm{i}$ \\
\hline Rh-105 & $1.18 \mathrm{E}+04$ & $1.40 \mathrm{E}-02$ & $8.44 E+05$ & d \\
\hline $\mathrm{Rh}-106 \mathrm{~m}$ & $1.96 \mathrm{E}+03$ & $1.46 \mathrm{E}-04$ & $1.34 \mathrm{E}+07$ & d \\
\hline Rh-107 & $1.12 E+05$ & $1.38 \mathrm{E}-03$ & $8.10 E+07$ & d \\
\hline Pd-100 & $2.80 \mathrm{E}+03$ & $7.78 \mathrm{E}-03$ & $3.60 \mathrm{E}+05$ & f \\
\hline Pd-101 & $7.40 \mathrm{E}+03$ & $1.97 \mathrm{E}-03$ & $3.75 E+06$ & $d$ \\
\hline Pd-103 & $6.20 E+03$ & $8.29 \mathrm{E}-02$ & $7.48 \mathrm{E}+04$ & f \\
\hline Pd-107 & $4.20 \mathrm{E}+03$ & $8.17 E+06$ & $5.14 \mathrm{E}-04$ & $\mathrm{i}$ \\
\hline Pd-109 & $3.00 \mathrm{E}+04$ & $1.40 \mathrm{E}-02$ & $2.14 \mathrm{E}+06$ & f \\
\hline $\mathrm{Ag}-102$ & $1.76 \mathrm{E}+04$ & $1.23 \mathrm{E}-04$ & $1.43 E+08$ & d \\
\hline Ag-103 & $3.00 \mathrm{E}+04$ & $1.08 \mathrm{E}-03$ & $2.78 E+07$ & $\mathrm{~d}$ \\
\hline $\mathrm{Ag}-104 \mathrm{~m}$ & $2.20 \mathrm{E}+04$ & $4.08 \mathrm{E}-04$ & $5.40 E+07$ & $\mathrm{~d}$ \\
\hline Ag-104 & $5.80 \mathrm{E}+04$ & $2.22 \mathrm{E}-03$ & $2.61 \mathrm{E}+07$ & $\mathrm{~d}$ \\
\hline Ag-105 & $1.64 \mathrm{E}+03$ & 5.41E-02 & $3.03 E+04$ & $\mathrm{~d}$ \\
\hline $\mathrm{Ag}-106 \mathrm{~m}$ & $2.80 \mathrm{E}+02$ & $1.91 \mathrm{E}-03$ & $1.47 \mathrm{E}+05$ & $\mathrm{~d}$ \\
\hline Ag-106 & $4.20 \mathrm{E}+04$ & $5.67 \mathrm{E}-04$ & $7.40 \mathrm{E}+07$ & $\mathrm{~d}$ \\
\hline $\mathrm{Ag}-108 \mathrm{~m}$ & $2.00 \mathrm{E}+02$ & $7.67 \mathrm{E}+00$ & $2.61 \mathrm{E}+01$ & $\mathrm{i}$ \\
\hline $\mathrm{Ag}-110 \mathrm{~m}$ & $2.60 \mathrm{E}+02$ & $5.50 \mathrm{E}-02$ & $4.75 E+03$ & $d$ \\
\hline Ag-111 & $1.44 \mathrm{E}+03$ & $9.12 \mathrm{E}-03$ & $1.58 E+05$ & f \\
\hline $\mathrm{Ag}-112$ & $7.60 \mathrm{E}+03$ & 8.47E-04 & $8.97 E+06$ & $d$ \\
\hline Ag-115 & $1.42 E+05$ & $1.74 \mathrm{E}-05$ & $8.18 E+09$ & d \\
\hline Cd-104 & $5.40 \mathrm{E}+04$ & $1.72 \mathrm{E}-03$ & $3.14 \mathrm{E}+07$ & d \\
\hline \multirow[t]{2}{*}{ Cd-107 } & $3.20 E+05$ & $7.09 \mathrm{E}-02$ & $4.51 E+06$ & d \\
\hline & \multicolumn{4}{|c|}{ Continued on next pag } \\
\hline
\end{tabular}


Table-Continued

\begin{tabular}{|c|c|c|c|c|}
\hline $\begin{array}{c}\text { ICRP-30 } \\
\text { Radionuclide }\end{array}$ & $\begin{array}{c}\text { Category } 3 \\
\text { Threshold } \\
\text { (Curies) }\end{array}$ & $\begin{array}{c}\text { Category } 3 \\
\text { Threshold (Grams) }\end{array}$ & $\begin{array}{c}\text { Calculated SA } \\
(\mathrm{Ci} / \mathrm{gm})^{5}\end{array}$ & Pathway \\
\hline Cd-109 & $1.80 \mathrm{E}+02$ & $7.00 \mathrm{E}-02$ & $2.58 \mathrm{E}+03$ & f \\
\hline $\mathrm{Cd}-113 \mathrm{~m}$ & $1.18 \mathrm{E}+01$ & $5.07 \mathrm{E}-02$ & $2.33 E+02$ & f \\
\hline Cd-113 & $1.10 \mathrm{E}+01$ & $3.20 \mathrm{E}+13$ & $3.40 \mathrm{E}-13$ & $\mathrm{f}$ \\
\hline $\mathrm{Cd}-115 \mathrm{~m}$ & $2.20 \mathrm{E}+02$ & 8.64E-03 & $2.55 \mathrm{E}+04$ & $\mathrm{f}$ \\
\hline Cd-115 & $2.20 \mathrm{E}+03$ & $4.31 \mathrm{E}-03$ & $5.10 \mathrm{E}+05$ & d \\
\hline Cd-117m & $1.40 \mathrm{E}+03$ & $1.76 \mathrm{E}-04$ & $7.97 E+06$ & $d$ \\
\hline Cd-117 & $5.60 \mathrm{E}+03$ & $5.20 \mathrm{E}-04$ & $1.08 \mathrm{E}+07$ & d \\
\hline In-109 & $9.00 \mathrm{E}+03$ & $1.31 \mathrm{E}-03$ & $6.85 E+06$ & d \\
\hline $\begin{array}{l}\text { In-110 } \\
(69.1 \mathrm{~min})\end{array}$ & $1.36 \mathrm{E}+04$ & $5.50 \mathrm{E}-04$ & $2.47 \mathrm{E}+07$ & d \\
\hline $\begin{array}{l}\text { In-110 } \\
(4.9 \mathrm{hr})\end{array}$ & $9.00 \mathrm{E}+02$ & $1.55 \mathrm{E}-04$ & $5.82 \mathrm{E}+06$ & $\mathrm{~d}$ \\
\hline In-111 & $2.20 E+03$ & $5.29 \mathrm{E}-03$ & $4.16 E+05$ & $\mathrm{~d}$ \\
\hline In- 112 & $1.18 E+06$ & $1.01 \mathrm{E}-02$ & $1.17 \mathrm{E}+08$ & $\mathrm{~d}$ \\
\hline In-113m & $3.00 \mathrm{E}+04$ & $1.79 \mathrm{E}-03$ & $1.67 \mathrm{E}+07$ & $d$ \\
\hline In- $114 \mathrm{~m}$ & $2.20 \mathrm{E}+02$ & $9.50 \mathrm{E}-03$ & $2.31 E+04$ & $\mathrm{f}$ \\
\hline In-115m & $1.78 \mathrm{E}+04$ & $2.93 \mathrm{E}-03$ & $6.08 E+06$ & $d$ \\
\hline In-115 & $1.04 \mathrm{E}+01$ & $1.71 \mathrm{E}+13$ & $6.10 \mathrm{E}-13$ & $\mathrm{i}$ \\
\hline In-116m & $6.40 \mathrm{E}+03$ & $2.14 \mathrm{E}-04$ & $2.99 E+07$ & d \\
\hline In-117m & $1.04 E+04$ & $7.54 \mathrm{E}-04$ & $1.38 \mathrm{E}+07$ & $d$ \\
\hline In-117 & $2.40 E+04$ & $6.54 \mathrm{E}-04$ & $3.67 \mathrm{E}+07$ & d \\
\hline In-119m & $1.04 \mathrm{E}+06$ & $1.18 \mathrm{E}-02$ & $8.78 E+07$ & $\mathrm{i}$ \\
\hline Sn-110 & $1.12 \mathrm{E}+04$ & $1.57 \mathrm{E}-03$ & $7.12 E+06$ & d \\
\hline Sn-111 & $1.68 \mathrm{E}+05$ & $3.50 \mathrm{E}-03$ & $4.80 \mathrm{E}+07$ & d \\
\hline Sn-113 & $1.30 \mathrm{E}+03$ & $1.30 \mathrm{E}-01$ & $1.00 \mathrm{E}+04$ & f \\
\hline $\mathrm{Sn}-117 \mathrm{~m}$ & $2.20 \mathrm{E}+03$ & $2.68 \mathrm{E}-02$ & $8.20 \mathrm{E}+04$ & f \\
\hline Sn-119m & $1.86 \mathrm{E}+03$ & 4.97E-01 & $3.75 E+03$ & f \\
\hline $\mathrm{Sn}-121 \mathrm{~m}$ & $1.78 \mathrm{E}+03$ & $3.31 \mathrm{E}+01$ & $5.37 \mathrm{E}+01$ & f \\
\hline Sn-121 & $4.60 \mathrm{E}+04$ & $4.81 \mathrm{E}-02$ & $9.57 E+05$ & $\mathrm{f}$ \\
\hline Sn-123m & $1.30 \mathrm{E}+05$ & $3.41 \mathrm{E}-03$ & $3.81 \mathrm{E}+07$ & d \\
\hline Sn-123 & $3.20 \mathrm{E}+02$ & $3.90 \mathrm{E}-02$ & $8.22 E+03$ & f \\
\hline Sn-125 & $5.60 \mathrm{E}+02$ & $5.17 \mathrm{E}-03$ & $1.08 \mathrm{E}+05$ & f \\
\hline Sn-126 & $1.70 \mathrm{E}+02$ & $6.00 \mathrm{E}+03$ & $2.84 \mathrm{E}-02$ & f \\
\hline Sn- 127 & $4.00 \mathrm{E}+03$ & $3.40 \mathrm{E}-04$ & $1.18 \mathrm{E}+07$ & d \\
\hline
\end{tabular}


Table-Continued

\begin{tabular}{|c|c|c|c|c|}
\hline $\begin{array}{l}\text { ICRP-30 } \\
\text { Radionuclide }\end{array}$ & $\begin{array}{c}\text { Category } 3 \\
\text { Threshold } \\
\text { (Curies) }\end{array}$ & $\begin{array}{c}\text { Category } 3 \\
\text { Threshold (Grams) }\end{array}$ & $\begin{array}{l}\text { Calculated SA } \\
(\mathrm{Ci} / \mathrm{gm})^{5}\end{array}$ & Pathway \\
\hline Sn-128 & $2.20 \mathrm{E}+04$ & 8.85E-04 & $2.49 \mathrm{E}+07$ & d \\
\hline $\mathrm{Sb}-115$ & $4.40 \mathrm{E}+04$ & $8.56 \mathrm{E}-04$ & $5.14 \mathrm{E}+07$ & d \\
\hline $\mathrm{Sb}-116 \mathrm{~m}$ & $4.20 \mathrm{E}+03$ & $1.56 \mathrm{E}-04$ & $2.69 E+07$ & d \\
\hline $\mathrm{Sb}-116$ & $2.60 \mathrm{E}+04$ & 2.53E-04 & $1.03 E+08$ & d \\
\hline Sb-117 & $3.00 \mathrm{E}+04$ & $3.14 \mathrm{E}-03$ & $9.57 \mathrm{E}+06$ & d \\
\hline$S b-118 m$ & $9.80 \mathrm{E}+02$ & $1.84 \mathrm{E}-04$ & $5.31 \mathrm{E}+06$ & d \\
\hline$S b-119$ & $5.80 \mathrm{E}+04$ & $8.39 \mathrm{E}-02$ & $6.91 E+05$ & $\mathrm{f}$ \\
\hline $\begin{array}{l}\text { Sb-120 } \\
(16 \mathrm{~min})\end{array}$ & $2.20 \mathrm{E}+06$ & $2.23 \mathrm{E}-02$ & $9.86 \mathrm{E}+07$ & $\mathrm{~d}$ \\
\hline $\begin{array}{l}\text { Sb } 120 \\
\text { (5.76 day) }\end{array}$ & $3.20 \mathrm{E}+02$ & $1.69 \mathrm{E}-03$ & $1.89 E+05$ & $\mathrm{~d}$ \\
\hline $\mathrm{Sb}-122$ & $1.86 \mathrm{E}+03$ & $4.69 \mathrm{E}-03$ & $3.96 \mathrm{E}+05$ & $d$ \\
\hline $\mathrm{Sb}-124 \mathrm{~m}$ & $6.20 \mathrm{E}+06$ & $8.26 \mathrm{E}-02$ & $7.51 \mathrm{E}+07$ & $\mathrm{i}$ \\
\hline$S b-124$ & $3.60 \mathrm{E}+02$ & $2.10 \mathrm{E}-02$ & $1.75 E+04$ & $\mathrm{f}$ \\
\hline Sb- 125 & $1.20 \mathrm{E}+03$ & $1.16 \mathrm{E}+00$ & $1.03 E+03$ & $\mathrm{f}$ \\
\hline $\mathrm{Sb}-126 \mathrm{~m}$ & $2.40 \mathrm{E}+04$ & $3.06 \mathrm{E}-04$ & $7.85 E+07$ & d \\
\hline $\mathrm{Sb}-126$ & $2.80 \mathrm{E}+02$ & $3.35 \mathrm{E}-03$ & $8.36 E+04$ & d \\
\hline $\mathrm{Sb}-127$ & $1.32 \mathrm{E}+03$ & 4.94E-03 & $2.67 E+05$ & d \\
\hline $\begin{array}{l}\mathrm{Sb}-128 \\
(10.4 \mathrm{~min})\end{array}$ & $3.80 \mathrm{E}+04$ & 2.69E-04 & $1.41 E+08$ & d \\
\hline $\begin{array}{l}\mathrm{Sb}-128 \\
\quad(9.01 \mathrm{hr})\end{array}$ & $5.60 \mathrm{E}+02$ & $2.06 \mathrm{E}-04$ & $2.72 E+06$ & d \\
\hline Sb-129 & $2.20 \mathrm{E}+03$ & $3.91 \mathrm{E}-04$ & $5.63 E+06$ & $d$ \\
\hline $\mathrm{Sb}-130$ & $6.80 \mathrm{E}+03$ & $1.88 \mathrm{E}-04$ & $3.62 \mathrm{E}+07$ & d \\
\hline Sb-131 & $2.40 \mathrm{E}+04$ & $3.85 \mathrm{E}-04$ & $6.24 E+07$ & d \\
\hline Te-116 & $8.00 E+04$ & 7.37E-03 & $1.09 E+07$ & d \\
\hline $\mathrm{Te}-121 \mathrm{~m}$ & $3.20 \mathrm{E}+02$ & $4.57 \mathrm{E}-02$ & $7.01 \mathrm{E}+03$ & f \\
\hline $\mathrm{Te}-121$ & $1.34 \mathrm{E}+03$ & $2.11 \mathrm{E}-02$ & $6.35 E+04$ & d \\
\hline $\mathrm{Te}-123 \mathrm{~m}$ & $4.00 \mathrm{E}+02$ & 4.49E-02 & $8.91 E+03$ & f \\
\hline $\mathrm{Te}-123$ & $3.00 \mathrm{E}+02$ & $1.03 E+12$ & $2.91 \mathrm{E}-10$ & $\mathrm{f}$ \\
\hline $\mathrm{Te}-125 \mathrm{~m}$ & $7.20 \mathrm{E}+02$ & $4.00 \mathrm{E}-02$ & $1.80 E+04$ & $\mathrm{f}$ \\
\hline $\mathrm{Te}-127 \mathrm{~m}$ & $4.00 \mathrm{E}+02$ & 4.24E-02 & $9.43 E+03$ & $\mathrm{f}$ \\
\hline Te-127 & $1.44 \mathrm{E}+05$ & $5.46 \mathrm{E}-02$ & $2.64 E+06$ & $\mathrm{f}$ \\
\hline $\mathrm{Te}-129 \mathrm{~m}$ & $4.00 \mathrm{E}+02$ & $1.30 \mathrm{E}-02$ & $3.01 E+04$ & $\mathrm{f}$ \\
\hline
\end{tabular}


Table-Continued

\begin{tabular}{|c|c|c|c|c|}
\hline $\begin{array}{c}\text { ICRP-30 } \\
\text { Radionuclide }\end{array}$ & $\begin{array}{c}\text { Category } 3 \\
\text { Threshold } \\
\text { (Curies) }\end{array}$ & $\begin{array}{c}\text { Category } 3 \\
\text { Threshold (Grams) }\end{array}$ & $\begin{array}{c}\text { Calculated SA } \\
(\mathrm{Ci} / \mathrm{gm})^{5}\end{array}$ & Pathway \\
\hline Te-129 & $2.20 \mathrm{E}+05$ & $1.05 \mathrm{E}-02$ & $2.09 \mathrm{E}+07$ & d \\
\hline $\mathrm{Te}-131 \mathrm{~m}$ & $8.00 \mathrm{E}+02$ & $1.00 \mathrm{E}-03$ & $7.98 \mathrm{E}+05$ & d \\
\hline $\mathrm{Te}-131$ & $5.20 \mathrm{E}+04$ & $9.06 \mathrm{E}-04$ & $5.74 \mathrm{E}+07$ & $\mathbf{i}$ \\
\hline $\mathrm{Te}-132$ & $6.00 \mathrm{E}+02$ & $1.98 \mathrm{E}-03$ & $3.04 \mathrm{E}+05$ & $\mathrm{f}$ \\
\hline Te-133m & $5.20 \mathrm{E}+04$ & $2.04 \mathrm{E}-03$ & $2.55 \mathrm{E}+07$ & $\mathrm{i}$ \\
\hline Te-133 & $6.80 \mathrm{E}+04$ & $5.99 \mathrm{E}-04$ & $1.14 \mathrm{E}+08$ & d \\
\hline $\mathrm{Te}-134$ & $2.00 \mathrm{E}+04$ & $5.96 \mathrm{E}-04$ & $3.36 \mathrm{E}+07$ & d \\
\hline $\mathrm{I}-120 \mathrm{~m}$ & $4.20 \mathrm{E}+03$ & $1.42 \mathrm{E}-04$ & $2.96 \mathrm{E}+07$ & $\mathrm{i}$ \\
\hline $\mathrm{I}-120$ & $1.86 \mathrm{E}+03$ & $9.62 \mathrm{E}-05$ & $1.93 \mathrm{E}+07$ & $\mathrm{i}$ \\
\hline $\mathrm{I}-121$ & $4.20 \mathrm{E}+03$ & $3.44 \mathrm{E}-04$ & $1.22 \mathrm{E}+07$ & $\mathrm{i}$ \\
\hline $\mathrm{I}-123$ & $9.00 \mathrm{E}+02$ & 4.66E-04 & $1.93 E+06$ & $\mathrm{f}$ \\
\hline I- 124 & $2.40 \mathrm{E}+00$ & $9.53 \mathrm{E}-06$ & $2.52 E+05$ & f \\
\hline I- 125 & $5.60 \mathrm{E}-01$ & $3.20 \mathrm{E}-05$ & $1.74 \mathrm{E}+04$ & $\mathrm{f}$ \\
\hline I- 126 & $4.60 \mathrm{E}-01$ & $5.78 \mathrm{E}-06$ & $7.96 \mathrm{E}+04$ & $\mathrm{f}$ \\
\hline I- 128 & $2.00 \mathrm{E}+04$ & $3.40 \mathrm{E}-04$ & $5.88 \mathrm{E}+07$ & i \\
\hline I-129 & $6.00 \mathrm{E}-02$ & $3.40 \mathrm{E}+02$ & $1.76 \mathrm{E}-04$ & $\mathrm{f}$ \\
\hline I-130 & $1.26 \mathrm{E}+02$ & $6.46 \mathrm{E}-05$ & $1.95 E+06$ & f \\
\hline $\mathrm{I}-131$ & $9.20 \mathrm{E}-01$ & $7.40 \mathrm{E}-06$ & $1.24 \mathrm{E}+05$ & $\mathbf{f}$ \\
\hline $\mathrm{I}-132 \mathrm{~m}$ & $1.66 \mathrm{E}+03$ & $9.74 \mathrm{E}-05$ & $1.70 \mathrm{E}+07$ & i \\
\hline I- 132 & $1.66 E+03$ & $1.61 \mathrm{E}-04$ & $1.03 E+07$ & $\mathrm{i}$ \\
\hline $\mathrm{I}-133$ & $1.94 \mathrm{E}+01$ & $1.71 \mathrm{E}-05$ & $1.13 E+06$ & f \\
\hline $\mathrm{I}-134$ & $5.80 \mathrm{E}+03$ & $2.17 \mathrm{E}-04$ & $2.67 \mathrm{E}+07$ & d \\
\hline I-135 & $4.20 \mathrm{E}+02$ & $1.20 \mathrm{E}-04$ & $3.51 E+06$ & $\mathrm{i}$ \\
\hline $\mathrm{Xe}-120$ & $2.00 \mathrm{E}+03$ & $5.11 \mathrm{E}-05$ & $3.92 E+07$ & d \\
\hline $\mathrm{Xe}-121$ & $4.00 \mathrm{E}+02$ & $1.03 \mathrm{E}-05$ & $3.88 \mathrm{E}+07$ & d \\
\hline $\mathrm{Xe}-122$ & $1.40 \mathrm{E}+04$ & $1.09 \mathrm{E}-02$ & $1.28 \mathrm{E}+06$ & d \\
\hline $\mathrm{Xe}-123$ & $1.20 \mathrm{E}+03$ & $9.79 \mathrm{E}-05$ & $1.23 E+07$ & d \\
\hline $\mathrm{Xe}-125$ & $4.00 \mathrm{E}+03$ & $2.71 \mathrm{E}-03$ & $1.47 E+06$ & d \\
\hline $\mathrm{Xe}-127$ & $2.00 \mathrm{E}+03$ & $7.08 \mathrm{E}-02$ & $2.82 E+04$ & d \\
\hline $\mathrm{Xe}-129 \mathrm{~m}$ & $4.00 \mathrm{E}+04$ & $3.16 \mathrm{E}-01$ & $1.27 \mathrm{E}+05$ & d \\
\hline $\mathrm{Xe}-131 \mathrm{~m}$ & $8.00 E+04$ & $9.55 \mathrm{E}-01$ & $8.38 E+04$ & d \\
\hline $\mathrm{Xe}-133 \mathrm{~m}$ & $2.00 E+04$ & $4.46 \mathrm{E}-02$ & $4.49 E+05$ & d \\
\hline $\mathrm{Xe}-133$ & $2.00 \mathrm{E}+04$ & $1.10 \mathrm{E}-01$ & $1.87 \mathrm{E}+05$ & d \\
\hline $\mathrm{Xe}-135 \mathrm{~m}$ & $1.80 \mathrm{E}+03$ & $1.98 \mathrm{E}-05$ & $9.11 E+07$ & d \\
\hline
\end{tabular}


Table-Continued

\begin{tabular}{|c|c|c|c|c|}
\hline $\begin{array}{c}\text { ICRP-30 } \\
\text { Radionuclide }\end{array}$ & $\begin{array}{c}\text { Category } 3 \\
\text { Threshold } \\
\text { (Curies) }\end{array}$ & $\begin{array}{c}\text { Category } 3 \\
\text { Threshold (Grams) }\end{array}$ & $\begin{array}{l}\text { Calculated SA } \\
(\mathrm{Ci} / \mathrm{gm})^{5}\end{array}$ & Pathway \\
\hline $\mathrm{Xe}-135$ & $2.00 E+03$ & $7.83 \mathrm{E}-04$ & $2.55 E+06$ & d \\
\hline $\mathrm{Xe}-138$ & $8.00 \mathrm{E}+02$ & 8.32E-06 & $9.62 E+07$ & d \\
\hline Cs- 125 & $6.20 \mathrm{E}+04$ & $1.85 \mathrm{E}-03$ & $3.34 \mathrm{E}+07$ & d \\
\hline Cs- 127 & $6.60 E+03$ & $1.67 \mathrm{E}-03$ & $3.95 E+06$ & d \\
\hline Cs- 129 & $3.80 \mathrm{E}+03$ & $5.01 \mathrm{E}-03$ & $7.58 \mathrm{E}+05$ & d \\
\hline Cs -130 & $6.00 \mathrm{E}+05$ & $1.24 \mathrm{E}-02$ & $4.84 \mathrm{E}+07$ & d \\
\hline Cs-131 & $2.80 \mathrm{E}+04$ & $2.72 \mathrm{E}-01$ & $1.03 E+05$ & f \\
\hline Cs- 132 & $1.14 \mathrm{E}+03$ & $7.46 \mathrm{E}-03$ & $1.53 E+05$ & d \\
\hline Cs-134m & $2.60 \mathrm{E}+05$ & $3.22 \mathrm{E}-02$ & $8.07 E+06$ & d \\
\hline Cs- 134 & $4.20 \mathrm{E}+01$ & $3.30 \mathrm{E}-02$ & $1.29 \mathrm{E}+03$ & $\mathrm{f}$ \\
\hline Cs-135m & $8.60 E+03$ & $3.27 \mathrm{E}-04$ & $2.63 E+07$ & d \\
\hline Cs-135 & $4.20 \mathrm{E}+02$ & $3.65 E+05$ & $1.15 E-03$ & f \\
\hline Cs- 136 & $4.00 \mathrm{E}+02$ & $5.46 \mathrm{E}-03$ & $7.33 E+04$ & d \\
\hline Cs- 137 & $6.00 \mathrm{E}+01$ & $6.90 \mathrm{E}-01$ & $8.70 \mathrm{E}+01$ & f \\
\hline Cs- 138 & $1.00 \mathrm{E}+04$ & $2.36 \mathrm{E}-04$ & $4.23 E+07$ & $\mathrm{~d}$ \\
\hline $\mathrm{Ba}-126$ & $2.00 \mathrm{E}+04$ & $1.29 \mathrm{E}-03$ & $1.55 \mathrm{E}+07$ & d \\
\hline $\mathrm{Ba}-128$ & $1.90 \mathrm{E}+03$ & $4.53 E-03$ & $4.20 E+05$ & f \\
\hline $\mathrm{Ba}-131 \mathrm{~m}$ & $8.20 \mathrm{E}+05$ & 8.34E-03 & $9.83 E+07$ & $d$ \\
\hline Ba-131 & $1.86 \mathrm{E}+03$ & $2.20 \mathrm{E}-02$ & $8.45 \mathrm{E}+04$ & d \\
\hline $\mathrm{Ba}-133 \mathrm{~m}$ & $1.08 \mathrm{E}+04$ & $1.78 \mathrm{E}-02$ & $6.06 E+05$ & $\mathrm{f}$ \\
\hline Ba-133 & $1.10 \mathrm{E}+03$ & $4.30 \mathrm{E}+00$ & $2.50 \mathrm{E}+02$ & f \\
\hline Ba-135m & $2.20 \mathrm{E}+04$ & $2.72 \mathrm{E}-02$ & $8.09 E+05$ & d \\
\hline Ba-139 & $1.98 E+05$ & $1.21 \mathrm{E}-02$ & $1.64 E+07$ & $d$ \\
\hline Ba-140 & $6.00 E+02$ & $8.20 \mathrm{E}-03$ & $7.32 \mathrm{E}+04$ & $\mathrm{f}$ \\
\hline Ba-141 & $7.20 \mathrm{E}+04$ & $9.86 \mathrm{E}-04$ & $7.30 \mathrm{E}+07$ & d \\
\hline Ba-142 & $6.80 \mathrm{E}+04$ & $5.44 \mathrm{E}-04$ & $1.25 E+08$ & $\mathrm{~d}$ \\
\hline La-131 & $5.00 \mathrm{E}+04$ & $2.05 E-03$ & $2.43 E+07$ & d \\
\hline La-132 & $2.00 \mathrm{E}+03$ & 4.04E-04 & $4.95 E+06$ & d \\
\hline La-135 & $1.16 \mathrm{E}+05$ & $9.74 \mathrm{E}-02$ & $1.19 E+06$ & d \\
\hline La-137 & $6.20 \mathrm{E}+02$ & $1.42 \mathrm{E}+04$ & $4.35 \mathrm{E}-02$ & $\mathrm{i}$ \\
\hline La-138 & $4.20 \mathrm{E}+01$ & $2.19 E+09$ & $1.92 \mathrm{E}-08$ & $\mathbf{i}$ \\
\hline La-140 & $4.00 \mathrm{E}+02$ & 7.19E-04 & $5.56 \mathrm{E}+05$ & d \\
\hline La-141 & $9.40 \mathrm{E}+04$ & $1.66 \mathrm{E}-02$ & $5.66 \mathrm{E}+06$ & $\mathrm{i}$ \\
\hline La-142 & $4.60 \mathrm{E}+03$ & $3.21 \mathrm{E}-04$ & $1.43 E+07$ & d \\
\hline
\end{tabular}


Table-Continued

\begin{tabular}{|c|c|c|c|c|}
\hline $\begin{array}{c}\text { ICRP-30 } \\
\text { Radionuclide }\end{array}$ & $\begin{array}{c}\text { Category } 3 \\
\text { Threshold } \\
\text { (Curies) }\end{array}$ & $\begin{array}{c}\text { Category } 3 \\
\text { Threshold (Grams) }\end{array}$ & $\begin{array}{l}\text { Calculated SA } \\
(\mathrm{Ci} / \mathrm{gm})^{5}\end{array}$ & Pathway \\
\hline La-143 & $9.40 \mathrm{E}+05$ & $1.02 \mathrm{E}-02$ & $9.24 \mathrm{E}+07$ & $\mathrm{~d}, \mathbf{i}$ \\
\hline Ce-134 & $1.54 \mathrm{E}+03$ & 4.74E-03 & $3.25 E+05$ & $\mathrm{f}$ \\
\hline Ce-135 & $1.58 \mathrm{E}+03$ & $1.20 \mathrm{E}-03$ & $1.32 \mathrm{E}+06$ & d \\
\hline $\mathrm{Ce}-137 \mathrm{~m}$ & $1.22 \mathrm{E}+04$ & $1.83 \mathrm{E}-02$ & $6.65 \mathrm{E}+05$ & f \\
\hline Ce-137 & $1.38 \mathrm{E}+05$ & $5.43 \mathrm{E}-02$ & $2.54 \mathrm{E}+06$ & $\mathrm{~d}$ \\
\hline Ce-139 & $3.20 \mathrm{E}+03$ & 4.69E-01 & $6.82 \mathrm{E}+03$ & f \\
\hline Ce-141 & $1.00 \mathrm{E}+03$ & $3.50 \mathrm{E}-02$ & $2.85 \mathrm{E}+04$ & $\mathrm{i}$ \\
\hline Ce-143 & $3.80 \mathrm{E}+03$ & $5.72 \mathrm{E}-03$ & $6.64 \mathrm{E}+05$ & d \\
\hline Ce-144 & $1.00 \mathrm{E}+02$ & $3.10 \mathrm{E}-02$ & $3.19 E+03$ & i \\
\hline Pr-136 & $4.60 \mathrm{E}+04$ & $4.36 \mathrm{E}-04$ & $1.06 \mathrm{E}+08$ & $d$ \\
\hline $\operatorname{Pr}-137$ & $3.00 \mathrm{E}+05$ & $1.67 \mathrm{E}-02$ & $1.79 \mathrm{E}+07$ & $d$ \\
\hline Pr- $138 \mathrm{~m}$ & $2.80 \mathrm{E}+03$ & $2.59 \mathrm{E}-04$ & $1.08 \mathrm{E}+07$ & $d$ \\
\hline Pr-139 & $3.00 \mathrm{E}+04$ & $6.00 \mathrm{E}-03$ & $5.00 \mathrm{E}+06$ & d \\
\hline Pr-142m & $1.04 \mathrm{E}+06$ & $1.15 \mathrm{E}-02$ & $9.07 \mathrm{E}+07$ & $\mathrm{i}$ \\
\hline Pr-142 & $1.06 \mathrm{E}+04$ & $9.19 \mathrm{E}-03$ & $1.15 E+06$ & f \\
\hline Pr-143 & $1.04 \mathrm{E}+03$ & $1.54 \mathrm{E}-02$ & $6.73 E+04$ & f \\
\hline Pr-144 & $1.04 \mathrm{E}+06$ & $1.38 \mathrm{E}-02$ & $7.56 \mathrm{E}+07$ & $\mathrm{i}$ \\
\hline $\operatorname{Pr}-145$ & $8.20 \mathrm{E}+04$ & $2.27 \mathrm{E}-02$ & $3.62 E+06$ & $\mathrm{i}$ \\
\hline $\operatorname{Pr}-147$ & $9.20 \mathrm{E}+04$ & $9.78 \mathrm{E}-04$ & $9.41 \mathrm{E}+07$ & $d$ \\
\hline Nd-136 & $1.02 E+05$ & $3.74 \mathrm{E}-03$ & $2.73 E+07$ & d \\
\hline Nd-138 & $5.20 \mathrm{E}+04$ & $1.15 \mathrm{E}-02$ & $4.51 \mathrm{E}+06$ & $\mathrm{i}$ \\
\hline $\mathrm{Nd}-139 \mathrm{~m}$ & $2.20 \mathrm{E}+03$ & 5.37E-04 & $4.10 \mathrm{E}+06$ & $\mathrm{~d}$ \\
\hline Nd-139 & $2.60 \mathrm{E}+05$ & $5.71 \mathrm{E}-03$ & $4.56 \mathrm{E}+07$ & $d$ \\
\hline Nd-141 & $2.40 \mathrm{E}+05$ & $2.69 \mathrm{E}-02$ & $8.93 E+06$ & $d$ \\
\hline $\mathrm{Nd}-147$ & $1.28 \mathrm{E}+03$ & $1.58 \mathrm{E}-02$ & $8.09 E+04$ & $\mathrm{f}$ \\
\hline Nd-149 & $1.56 \mathrm{E}+04$ & $1.28 \mathrm{E}-03$ & $1.22 E+07$ & $d$ \\
\hline Nd-151 & $9.40 \mathrm{E}+04$ & $9.39 \mathrm{E}-04$ & $1.00 \mathrm{E}+08$ & $d$ \\
\hline Pm-141 & $5.40 \mathrm{E}+04$ & $8.46 \mathrm{E}-04$ & $6.38 \mathrm{E}+07$ & $d$ \\
\hline $\mathrm{Pm}-143$ & $2.60 \mathrm{E}+03$ & $7.54 \mathrm{E}-01$ & $3.45 E+03$ & d \\
\hline Pm-144 & $4.80 \mathrm{E}+02$ & $1.92 \mathrm{E}-01$ & $2.50 \mathrm{E}+03$ & d \\
\hline $\mathrm{Pm}-145$ & $2.00 \mathrm{E}+03$ & $1.40 \mathrm{E}+01$ & $1.39 \mathrm{E}+02$ & $\mathrm{i}$ \\
\hline Pm-146 & $4.20 \mathrm{E}+02$ & $9.49 \mathrm{E}-01$ & $4.43 E+02$ & $\mathrm{i}$ \\
\hline Pm-147 & $1.00 \mathrm{E}+03$ & $9.50 \mathrm{E}-01$ & $9.27 E+02$ & $\mathrm{i}$ \\
\hline $\mathrm{Pm}-148 \mathrm{~m}$ & $3.60 \mathrm{E}+02$ & $1.68 \mathrm{E}-02$ & $2.14 E+04$ & $d$ \\
\hline
\end{tabular}




\begin{tabular}{|c|c|c|c|c|}
\hline $\begin{array}{c}\text { ICRP-30 } \\
\text { Radionuclide }\end{array}$ & $\begin{array}{c}\text { Category } 3 \\
\text { Threshold } \\
\text { (Curies) }\end{array}$ & $\begin{array}{c}\text { Category } 3 \\
\text { Threshold (Grams) }\end{array}$ & $\begin{array}{c}\text { Calculated SA } \\
(\mathrm{Ci} / \mathrm{gm})^{5}\end{array}$ & Pathway \\
\hline $\mathrm{Pm}-148$ & $8.20 E+02$ & $4.99 \mathrm{E}-03$ & $1.64 \mathrm{E}+05$ & f \\
\hline Pm-149 & $4.20 \mathrm{E}+03$ & $1.06 \mathrm{E}-02$ & $3.96 \mathrm{E}+05$ & f \\
\hline $\mathrm{Pm}-150$ & $3.80 \mathrm{E}+03$ & 4.87E-04 & $7.80 \mathrm{E}+06$ & d \\
\hline Pm-151 & $4.20 \mathrm{E}+03$ & $5.74 \mathrm{E}-03$ & $7.31 E+05$ & d \\
\hline $\mathrm{Sm}-141 \mathrm{~m}$ & $2.00 \mathrm{E}+04$ & 3.93E-04 & $5.08 \mathrm{E}+07$ & d \\
\hline $\mathrm{Sm}-141$ & $6.80 E+04$ & $5.20 \mathrm{E}-04$ & $1.31 E+08$ & d \\
\hline Sm-142 & $1.62 E+05$ & 8.87E-03 & $1.83 E+07$ & d \\
\hline Sm-145 & $3.60 \mathrm{E}+03$ & $1.36 \mathrm{E}+\infty 0$ & $2.65 E+03$ & f \\
\hline Sm-146 & $4.20 \mathrm{E}-01$ & $1.77 \mathrm{E}+04$ & $2.38 \mathrm{E}-05$ & $\mathrm{i}$ \\
\hline Sm-147 & $4.20 \mathrm{E}-01$ & $1.83 E+07$ & $2.30 \mathrm{E}-08$ & i \\
\hline Sm-151 & $1.00 \mathrm{E}+03$ & $3.80 \mathrm{E}+01$ & $2.63 \mathrm{E}+01$ & $\mathbf{i}$ \\
\hline Sm-153 & $9.20 \mathrm{E}+03$ & $2.10 \mathrm{E}-02$ & $4.39 \mathrm{E}+05$ & f \\
\hline $\mathrm{Sm}-155$ & $3.60 E+05$ & $6.56 \mathrm{E}-03$ & $5.49 \mathrm{E}+07$ & d \\
\hline Sm-156 & $1.40 \mathrm{E}+04$ & $6.55 \mathrm{E}-03$ & $2.14 E+06$ & d \\
\hline Eu-145 & $6.40 \mathrm{E}+02$ & $4.22 \mathrm{E}-03$ & $1.52 \mathrm{E}+05$ & d \\
\hline Eu-146 & $4.00 \mathrm{E}+02$ & $2.06 \mathrm{E}-03$ & $1.94 \mathrm{E}+05$ & d \\
\hline Eu-147 & $1.76 \mathrm{E}+03$ & 4.76E-02 & $3.70 \mathrm{E}+04$ & d \\
\hline Eu-148 & $3.80 \mathrm{E}+02$ & $2.35 \mathrm{E}-02$ & $1.62 E+04$ & d \\
\hline Eu-149 & $6.80 \mathrm{E}+03$ & $7.22 \mathrm{E}-01$ & $9.41 E+03$ & f \\
\hline $\begin{array}{l}\mathrm{Eu}-150 \\
(12.6 \mathrm{hr})\end{array}$ & $4.00 \mathrm{E}+04$ & $2.41 \mathrm{E}-02$ & $1.66 \mathrm{E}+06$ & $d$ \\
\hline $\begin{array}{l}\mathrm{Eu}-150 \\
(34.2 \mathrm{yr})\end{array}$ & $2.00 \mathrm{E}+02$ & $2.87 \mathrm{E}+00$ & $6.97 \mathrm{E}+01$ & $\mathrm{i}$ \\
\hline $\mathrm{Eu}-152 \mathrm{~m}$ & $5.60 E+03$ & $2.53 \mathrm{E}-03$ & $2.21 \mathrm{E}+06$ & d \\
\hline Eu-152 & $2.00 \mathrm{E}+02$ & $1.20 \mathrm{E}+00$ & $1.77 \mathrm{E}+02$ & i \\
\hline Eu-154 & $2.00 E+02$ & 7.60E-01 & $2.64 \mathrm{E}+02$ & $\mathrm{i}$ \\
\hline Eu-155 & $9.40 \mathrm{E}+02$ & $2.00 \mathrm{E}+00$ & $4.65 E+02$ & $\mathrm{i}$ \\
\hline Eu-156 & $4.60 \mathrm{E}+02$ & 8.35E-03 & $5.51 E+04$ & d \\
\hline Eu-157 & $9.80 \mathrm{E}+02$ & $7.44 \mathrm{E}-04$ & $1.32 \mathrm{E}+06$ & d \\
\hline Eu-158 & $2.00 \mathrm{E}+04$ & $7.71 \mathrm{E}-04$ & $2.59 E+07$ & d \\
\hline Gd-145 & $1.66 \mathrm{E}+04$ & 2.93E-04 & $5.66 \mathrm{E}+07$ & d \\
\hline Gd-146 & $7.40 \mathrm{E}+02$ & $4.00 \mathrm{E}-02$ & $1.85 E+04$ & f \\
\hline Gd-147 & $8.60 E+02$ & $1.54 \mathrm{E}-03$ & $5.60 \mathrm{E}+05$ & d \\
\hline Gd-148 & $8.20 \mathrm{E}-02$ & $3.16 \mathrm{E}-03$ & $2.60 \mathrm{E}+01$ & $\mathrm{i}$ \\
\hline
\end{tabular}




\begin{tabular}{|c|c|c|c|c|}
\hline $\begin{array}{l}\text { ICRP-30 } \\
\text { Radionuclide }\end{array}$ & $\begin{array}{c}\text { Category } 3 \\
\text { Threshold } \\
\text { (Curies) }\end{array}$ & $\begin{array}{c}\text { Category } 3 \\
\text { Threshold (Grams) }\end{array}$ & $\begin{array}{l}\text { Calculated SA } \\
(\mathrm{Ci} / \mathrm{gm})^{5}\end{array}$ & Pathway \\
\hline Gd-149 & $2.20 \mathrm{E}+03$ & $2.36 \mathrm{E}-02$ & $9.32 E+04$ & d \\
\hline Gd-151 & $4.00 \mathrm{E}+03$ & $5.55 \mathrm{E}-01$ & $7.21 E+03$ & $\mathbf{f}$ \\
\hline Gd-152 & $1.04 \mathrm{E}-01$ & $4.77 \mathrm{E}+09$ & $2.18 \mathrm{E}-11$ & i \\
\hline Gd-153 & $1.00 E+03$ & $2.80 \mathrm{E}-01$ & $3.53 E+03$ & $\mathrm{i}$ \\
\hline Gd-159 & $2.80 \mathrm{E}+04$ & 2.64E-02 & $1.06 \mathrm{E}+06$ & d \\
\hline $\mathrm{Tb}-147$ & $3.80 \mathrm{E}+03$ & $2.94 \mathrm{E}-04$ & $1.29 \mathrm{E}+07$ & d \\
\hline $\mathrm{Tb}-149$ & $3.40 \mathrm{E}+03$ & $6.71 \mathrm{E}-04$ & $5.07 \mathrm{E}+06$ & d \\
\hline $\mathrm{Tb}-150$ & $4.40 \mathrm{E}+03$ & $6.88 \mathrm{E}-04$ & $6.39 E+06$ & d \\
\hline $\mathrm{Tb}-151$ & $1.70 \mathrm{E}+03$ & $1.44 \mathrm{E}-03$ & $1.18 \mathrm{E}+06$ & d \\
\hline $\mathrm{Tb}-153$ & $6.00 \mathrm{E}+03$ & $1.64 \mathrm{E}-02$ & $3.65 \mathrm{E}+05$ & d \\
\hline $\mathrm{Tb}-154$ & $6.40 \mathrm{E}+02$ & $6.73 E-04$ & $9.51 E+05$ & d \\
\hline $\mathrm{Tb}-155$ & $8.80 E+03$ & $5.56 \mathrm{E}-02$ & $1.58 \mathrm{E}+05$ & d \\
\hline $\begin{array}{r}\mathrm{Tb}-156 \mathrm{~m} \\
(5.0 \mathrm{hr})\end{array}$ & $3.20 \mathrm{E}+05$ & $7.96 \mathrm{E}-02$ & $4.02 E+06$ & $\mathrm{i}$ \\
\hline $\begin{array}{l}\mathrm{Tb}-156 \mathrm{~m} \\
(24.4 \mathrm{hr})\end{array}$ & $6.00 \mathrm{E}+04$ & $7.29 \mathrm{E}-02$ & $8.24 E+05$ & f \\
\hline $\mathrm{Tb}-156$ & $5.00 \mathrm{E}+02$ & $3.19 \mathrm{E}-03$ & $1.57 \mathrm{E}+05$ & d \\
\hline $\mathrm{Tb}-157$ & $3.20 \mathrm{E}+03$ & $2.11 E+02$ & $1.52 \mathrm{E}+01$ & i \\
\hline Tb-158 & $2.00 \mathrm{E}+02$ & $1.33 E+01$ & $1.51 \mathrm{E}+01$ & $\mathrm{i}$ \\
\hline Tb-160 & $5.60 \mathrm{E}+02$ & $5.00 \mathrm{E}-02$ & $1.13 E+04$ & $f$ \\
\hline $\mathrm{Tb}-161$ & $3.40 \mathrm{E}+03$ & $2.90 \mathrm{E}-02$ & $1.17 \mathrm{E}+05$ & f \\
\hline Dy-155 & $4.00 \mathrm{E}+03$ & $1.98 \mathrm{E}-03$ & $2.02 E+06$ & d \\
\hline Dy-157 & $5.60 \mathrm{E}+03$ & $2.27 \mathrm{E}-03$ & $2.46 \mathrm{E}+06$ & d \\
\hline Dy-159 & $2.40 \mathrm{E}+03$ & $4.22 \mathrm{E}-01$ & $5.69 E+03$ & d \\
\hline Dy-165 & $3.20 \mathrm{E}+05$ & $3.93 \mathrm{E}-02$ & $8.14 E+06$ & d \\
\hline Dy-166 & $1.74 \mathrm{E}+03$ & $7.52 \mathrm{E}-03$ & $2.31 E+05$ & f \\
\hline Ho- 155 & $1.56 \mathrm{E}+05$ & $6.17 \mathrm{E}-03$ & $2.53 E+07$ & d \\
\hline Ho- 157 & $1.82 E+05$ & $1.91 \mathrm{E}-03$ & $9.50 \mathrm{E}+07$ & d \\
\hline Ho- 159 & $1.08 E+05$ & $3.01 \mathrm{E}-03$ & $3.58 E+07$ & d \\
\hline Ho-161 & $9.00 \mathrm{E}+05$ & $1.16 \mathrm{E}-01$ & $7.79 \mathrm{E}+06$ & d \\
\hline Ho- $162 \mathrm{~m}$ & $2.20 \mathrm{E}+04$ & $1.29 \mathrm{E}-03$ & $1.71 E+07$ & d \\
\hline Ho-162 & $7.20 \mathrm{E}+05$ & $9.30 \mathrm{E}-03$ & $7.74 \mathrm{E}+07$ & d \\
\hline Ho- $164 \mathrm{~m}$ & $3.20 \mathrm{E}+06$ & $1.05 \mathrm{E}-01$ & $3.06 \mathrm{E}+07$ & i \\
\hline Ho-164 & $6.20 E+06$ & $1.57 \mathrm{E}-01$ & $3.95 E+07$ & $\mathrm{i}$ \\
\hline
\end{tabular}


Table-Continued

\begin{tabular}{|c|c|c|c|c|}
\hline $\begin{array}{c}\text { ICRP-30 } \\
\text { Radionuclide }\end{array}$ & $\begin{array}{c}\text { Category } 3 \\
\text { Threshold } \\
\text { (Curies) }\end{array}$ & $\begin{array}{c}\text { Category } 3 \\
\text { Threshold (Grams) }\end{array}$ & $\begin{array}{l}\text { Calculated SA } \\
(\mathrm{Ci} / \mathrm{gm})^{5}\end{array}$ & Pathway \\
\hline $\mathrm{Ho}-166 \mathrm{~m}$ & $7.20 \mathrm{E}+01$ & $4.00 \mathrm{E}+01$ & $1.80 \mathrm{E}+00$ & $\mathrm{i}$ \\
\hline Нo-166 & $6.80 \mathrm{E}+03$ & $9.65 \mathrm{E}-03$ & $7.05 E+05$ & f \\
\hline Ho-167 & $1.22 \mathrm{E}+04$ & 2.01E-03 & $6.06 \mathrm{E}+06$ & d \\
\hline Er-161 & $6.60 \mathrm{E}+03$ & $1.10 \mathrm{E}-03$ & $6.01 E+06$ & d \\
\hline Er-165 & $1.14 E+06$ & $6.22 \mathrm{E}-01$ & $1.83 E+06$ & f \\
\hline Er-169 & $4.20 \mathrm{E}+03$ & $5.05 \mathrm{E}-02$ & $8.31 E+04$ & f \\
\hline Er-171 & $5.40 \mathrm{E}+03$ & $2.22 \mathrm{E}-03$ & $2.44 \mathrm{E}+06$ & d \\
\hline Er-172 & $1.70 \mathrm{E}+03$ & $4.60 \mathrm{E}-03$ & $3.70 \mathrm{E}+05$ & d \\
\hline $\mathrm{Tm}-162$ & $4.20 \mathrm{E}+04$ & $7.85 \mathrm{E}-04$ & $5.35 \mathrm{E}+07$ & d \\
\hline Tm-166 & $9.20 \mathrm{E}+02$ & $3.75 \mathrm{E}-04$ & $2.45 \mathrm{E}+06$ & d \\
\hline Tm-167 & $2.80 \mathrm{E}+03$ & $3.31 \mathrm{E}-02$ & $8.46 E+04$ & f \\
\hline $\mathrm{Tm}-170$ & $5.20 \mathrm{E}+02$ & $8.70 \mathrm{E}-02$ & $5.97 \mathrm{E}+03$ & $\mathbf{f}$ \\
\hline Tm-171 & $3.20 \mathrm{E}+03$ & $2.94 \mathrm{E}+00$ & $1.09 E+03$ & i \\
\hline Tm-172 & $2.40 \mathrm{E}+03$ & $8.38 \mathrm{E}-03$ & $2.87 \mathrm{E}+05$ & $\mathrm{~d}, \mathrm{f}$ \\
\hline Tm-173 & $4.40 \mathrm{E}+03$ & $2.00 \mathrm{E}-03$ & $2.20 \mathrm{E}+06$ & $d$ \\
\hline Tm-175 & $5.80 \mathrm{E}+04$ & $8.20 \mathrm{E}-04$ & $7.07 \mathrm{E}+07$ & $\mathrm{~d}$ \\
\hline $\mathrm{Yb}-162$ & $4.40 \mathrm{E}+05$ & $7.16 \mathrm{E}-03$ & $6.14 E+07$ & $d$ \\
\hline$Y b-166$ & $8.40 \mathrm{E}+02$ & $2.52 \mathrm{E}-03$ & $3.33 E+05$ & $\mathrm{~d}$ \\
\hline Yb-167 & $3.00 \mathrm{E}+05$ & $4.66 \mathrm{E}-03$ & $6.43 E+07$ & $d$ \\
\hline Yb-169 & $1.64 \mathrm{E}+03$ & $6.79 \mathrm{E}-02$ & $2.41 \mathrm{E}+04$ & $\mathrm{f}$ \\
\hline$Y b-175$ & $7.40 \mathrm{E}+03$ & $4.16 \mathrm{E}-02$ & $1.78 \mathrm{E}+05$ & f \\
\hline$Y b-177$ & $4.40 \mathrm{E}+04$ & 4.72E- 03 & $9.32 E+06$ & $d$ \\
\hline Yb-178 & $4.20 \mathrm{E}+05$ & $2.94 \mathrm{E}-02$ & $1.43 E+07$ & i \\
\hline Lu-169 & $1.54 \mathrm{E}+03$ & $2.83 \mathrm{E}-03$ & $5.45 \mathrm{E}+05$ & d \\
\hline Lu-170 & $5.00 \mathrm{E}+02$ & $1.30 \mathrm{E}-03$ & $3.84 \mathrm{E}+05$ & d \\
\hline Lu-171 & $1.40 \mathrm{E}+03$ & $1.51 \mathrm{E}-02$ & $9.29 E+04$ & $d$ \\
\hline Lu-172 & $4.80 \mathrm{E}+02$ & $4.24 \mathrm{E}-03$ & $1.13 \mathrm{E}+05$ & d \\
\hline Lu-173 & $3.00 \mathrm{E}+03$ & $1.99 E+00$ & $1.51 \mathrm{E}+03$ & f \\
\hline $\mathrm{Lu}-174 \mathrm{~m}$ & $1.30 \mathrm{E}+03$ & $2.46 \mathrm{E}-01$ & $5.29 \mathrm{E}+03$ & $\mathrm{f}$ \\
\hline Lu-174 & $1.04 \mathrm{E}+03$ & $1.67 \mathrm{E}+00$ & $6.21 E+02$ & $\mathrm{i}$ \\
\hline $\mathrm{Lu}-176 \mathrm{~m}$ & $2.00 \mathrm{E}+05$ & 4.13E-02 & $4.84 E+06$ & i \\
\hline Lu-176 & $5.20 \mathrm{E}+01$ & $9.21 \mathrm{E}+08$ & $5.65 \mathrm{E}-08$ & $\mathbf{i}$ \\
\hline $\mathrm{Lu}-177 \mathrm{~m}$ & $4.40 \mathrm{E}+02$ & $9.60 \mathrm{E}-02$ & $4.59 E+03$ & f \\
\hline Lu-177 & $3.40 \mathrm{E}+03$ & $3.09 \mathrm{E}-02$ & $1.10 \mathrm{E}+05$ & f \\
\hline
\end{tabular}


Table-Continued

\begin{tabular}{|c|c|c|c|c|}
\hline $\begin{array}{c}\text { ICRP-30 } \\
\text { Radionuclide }\end{array}$ & $\begin{array}{c}\text { Category } 3 \\
\text { Threshold } \\
\text { (Curies) }\end{array}$ & $\begin{array}{c}\text { Category } 3 \\
\text { Threshold (Grams) }\end{array}$ & $\begin{array}{l}\text { Calculated SA } \\
(\mathrm{Ci} / \mathrm{gm})^{5}\end{array}$ & Pathway \\
\hline $\mathrm{Lu}-178 \mathrm{~m}$ & $7.20 \mathrm{E}+05$ & $1.55 \mathrm{E}-02$ & $4.65 E+07$ & d \\
\hline Lu-178 & $2.60 \mathrm{E}+05$ & $6.99 \mathrm{E}-03$ & $3.72 E+07$ & d \\
\hline Lu-179 & $1.02 E+05$ & $2.67 \mathrm{E}-02$ & $3.82 E+06$ & d \\
\hline Hf- 170 & $2.80 \mathrm{E}+03$ & $2.43 \mathrm{E}-03$ & $1.15 E+06$ & d \\
\hline Hf- 172 & $9.40 \mathrm{E}+01$ & $8.45 \mathrm{E}-02$ & $1.11 \mathrm{E}+03$ & $\mathrm{i}$ \\
\hline Hf- 173 & $3.20 \mathrm{E}+03$ & $4.24 \mathrm{E}-03$ & $7.55 E+05$ & d \\
\hline Hf -175 & $2.00 \mathrm{E}+03$ & $1.88 \mathrm{E}-01$ & $1.07 E+04$ & f \\
\hline Hf- $177 \mathrm{~m}$ & $6.20 \mathrm{E}+05$ & $3.00 \mathrm{E}-02$ & $2.07 \mathrm{E}+07$ & $\mathrm{i}$ \\
\hline Hf-178m & $1.04 \mathrm{E}+01$ & $1.60 \mathrm{E}-01$ & $6.48 \mathrm{E}+01$ & $\mathbf{i}$ \\
\hline Hf- $179 \mathrm{~m}$ & $3.20 \mathrm{E}+03$ & $1.10 \mathrm{E}-01$ & $2.91 E+04$ & $\mathbf{i}$ \\
\hline $\mathrm{Hf}-180 \mathrm{~m}$ & $2.40 \mathrm{E}+03$ & $7.58 \mathrm{E}-04$ & $3.17 \mathrm{E}+06$ & d \\
\hline Hf-181 & $7.60 \mathrm{E}+02$ & $4.50 \mathrm{E}-02$ & $1.70 E+04$ & f \\
\hline Hf $-182 \mathrm{~m}$ & $1.34 \mathrm{E}+04$ & $7.98 \mathrm{E}-04$ & $1.68 \mathrm{E}+07$ & $d$ \\
\hline Hf- 182 & $8.20 \mathrm{E}+00$ & $3.76 \mathrm{E}+04$ & $2.18 \mathrm{E}-04$ & $\mathrm{i}$ \\
\hline Hf -183 & $1.58 \mathrm{E}+04$ & $9.84 \mathrm{E}-04$ & $1.61 \mathrm{E}+07$ & d \\
\hline Hf- 184 & $1.34 \mathrm{E}+04$ & $3.24 \mathrm{E}-03$ & $4.13 E+06$ & $d$ \\
\hline Ta-172 & $1.58 \mathrm{E}+04$ & $5.32 \mathrm{E}-04$ & $2.97 \mathrm{E}+07$ & d \\
\hline Ta-173 & $9.80 \mathrm{E}+03$ & $1.97 \mathrm{E}-03$ & $4.96 \mathrm{E}+06$ & $d$ \\
\hline $\mathrm{Ta}-174$ & $1.92 \mathrm{E}+04$ & $1.28 \mathrm{E}-03$ & $1.50 \mathrm{E}+07$ & d \\
\hline Ta-175 & $3.80 \mathrm{E}+03$ & $2.23 \mathrm{E}-03$ & $1.71 E+06$ & $\mathrm{~d}$ \\
\hline Тa-176 & $1.20 \mathrm{E}+03$ & $5.44 \mathrm{E}-04$ & $2.20 \mathrm{E}+06$ & $d$ \\
\hline Тa-177 & $5.40 \mathrm{E}+04$ & $1.73 \mathrm{E}-01$ & $3.13 E+05$ & $d$ \\
\hline Тa-178 & $1.12 \mathrm{E}+06$ & $1.40 \mathrm{E}-01$ & $8.01 \mathrm{E}+06$ & d \\
\hline Тa-179 & $3.00 E+04$ & $2.73 E+01$ & $1.10 \mathrm{E}+03$ & w \\
\hline $\mathrm{Ta}-180 \mathrm{~m}$ & $3.20 \mathrm{E}+05$ & $1.49 \mathrm{E}-01$ & $2.15 E+06$ & d \\
\hline Ta-180 & $2.00 \mathrm{E}+03$ & $1.01 \mathrm{E}+13$ & $1.99 \mathrm{E}-10$ & $\mathrm{i}$ \\
\hline Ta-182m & $2.40 E+05$ & $3.68 \mathrm{E}-03$ & $6.52 \mathrm{E}+07$ & d \\
\hline Ta-182 & $6.20 \mathrm{E}+02$ & $9.94 \mathrm{E}-02$ & $6.24 E+03$ & d \\
\hline Ta-183 & $3.60 \mathrm{E}+03$ & $2.57 \mathrm{E}-02$ & $1.40 \mathrm{E}+05$ & d \\
\hline Ta-184 & $1.08 \mathrm{E}+03$ & $5.52 \mathrm{E}-04$ & $1.96 E+06$ & d \\
\hline Ta-185 & $1.34 \mathrm{E}+05$ & $6.46 \mathrm{E}-03$ & $2.07 \mathrm{E}+07$ & d \\
\hline Ta-186 & $5.40 \mathrm{E}+04$ & $5.61 E-04$ & $9.63 E+07$ & d \\
\hline W-176 & $4.20 \mathrm{E}+04$ & $5.42 \mathrm{E}-03$ & $7.74 \mathrm{E}+06$ & d \\
\hline W-177 & $1.14 \mathrm{E}+04$ & $1.45 \mathrm{E}-03$ & $7.87 E+06$ & d \\
\hline
\end{tabular}




\begin{tabular}{|c|c|c|c|c|}
\hline $\begin{array}{l}\text { ICRP-30 } \\
\text { Radionuclide }\end{array}$ & $\begin{array}{c}\text { Category } 3 \\
\text { Threshold } \\
\text { (Curies) }\end{array}$ & $\begin{array}{c}\text { Category } 3 \\
\text { Threshold (Grams) }\end{array}$ & $\begin{array}{l}\text { Calculated SA } \\
(\mathrm{Ci} / \mathrm{gm})^{5}\end{array}$ & Pathway \\
\hline W-178 & $4.60 \mathrm{E}+03$ & $1.36 \mathrm{E}-01$ & $3.38 \mathrm{E}+04$ & f \\
\hline W-179 & $2.00 \mathrm{E}+07$ & $7.14 \mathrm{E}-01$ & $2.80 \mathrm{E}+07$ & $\mathbf{i}$ \\
\hline W-181 & $1.30 \mathrm{E}+04$ & $2.18 \mathrm{E}+00$ & $5.95 \mathrm{E}+03$ & f \\
\hline W-185 & $1.38 \mathrm{E}+03$ & $1.47 \mathrm{E}-01$ & $9.40 \mathrm{E}+03$ & f \\
\hline W-187 & $2.20 \mathrm{E}+03$ & $3.14 \mathrm{E}-03$ & $7.01 E+05$ & d \\
\hline W-188 & $2.80 \mathrm{E}+02$ & $2.80 \mathrm{E}-02$ & $1.00 \mathrm{E}+04$ & f \\
\hline $\operatorname{Re}-177$ & $1.22 E+05$ & $1.61 \mathrm{E}-03$ & $7.59 \mathrm{E}+07$ & $\mathrm{~d}$ \\
\hline Re-178 & $9.20 \mathrm{E}+04$ & $1.15 \mathrm{E}-03$ & $8.00 \mathrm{E}+07$ & d \\
\hline $\operatorname{Re}-181$ & $2.20 \mathrm{E}+03$ & $2.54 \mathrm{E}-03$ & $8.66 \mathrm{E}+05$ & d \\
\hline $\begin{array}{l}\operatorname{Re}-182 \\
(12.7 \mathrm{hr})\end{array}$ & $1.38 \mathrm{E}+03$ & $1.02 \mathrm{E}-03$ & $1.36 \mathrm{E}+06$ & d \\
\hline $\begin{array}{l}\operatorname{Re}-182 \\
(64.0 \mathrm{hr})\end{array}$ & $5.00 \mathrm{E}+02$ & $1.86 \mathrm{E}-03$ & $2.69 \mathrm{E}+05$ & d \\
\hline $\operatorname{Re}-184 m$ & $1.22 \mathrm{E}+03$ & $2.84 \mathrm{E}-01$ & $4.30 \mathrm{E}+03$ & f \\
\hline $\operatorname{Re}-184$ & $9.00 \mathrm{E}+02$ & $4.82 \mathrm{E}-02$ & $1.87 E+04$ & $d$ \\
\hline $\operatorname{Re}-186 m$ & $5.60 \mathrm{E}+02$ & $5.82 \mathrm{E}+04$ & $9.61 E-03$ & $f$ \\
\hline $\operatorname{Re}-186$ & $5.00 \mathrm{E}+03$ & $2.69 \mathrm{E}-02$ & $1.86 \mathrm{E}+05$ & f \\
\hline $\operatorname{Re}-187$ & $3.40 \mathrm{E}+05$ & $8.89 E+12$ & 3.83E-08 & $\mathbf{f}$ \\
\hline $\operatorname{Re}-188 \mathrm{~m}$ & $1.04 \mathrm{E}+06$ & $1.93 \mathrm{E}-02$ & $5.38 E+07$ & $\mathrm{i}$ \\
\hline $\operatorname{Re}-188$ & $2.20 E+04$ & $2.24 \mathrm{E}-02$ & $9.82 E+05$ & $\mathrm{f}$ \\
\hline $\operatorname{Re}-189$ & $2.40 \mathrm{E}+04$ & $3.52 E-02$ & $6.82 E+05$ & d \\
\hline Os- 180 & $4.20 \mathrm{E}+06$ & $8.84 \mathrm{E}-02$ & $4.75 E+07$ & $\mathrm{i}$ \\
\hline Os-181 & $8.80 \mathrm{E}+03$ & $8.89 \mathrm{E}-04$ & $9.90 E+06$ & d \\
\hline Os-182 & $2.80 \mathrm{E}+03$ & $3.58 \mathrm{E}-03$ & $7.83 E+05$ & $\mathrm{~d}$ \\
\hline Os- 185 & $1.10 \mathrm{E}+03$ & $1.47 \mathrm{E}-01$ & $7.51 E+03$ & d \\
\hline Os- $189 \mathrm{~m}$ & $2.00 \mathrm{E}+06$ & $7.24 \mathrm{E}-01$ & $2.76 \mathrm{E}+06$ & i \\
\hline Os-191m & $1.50 \mathrm{E}+05$ & $1.19 \mathrm{E}-01$ & $1.26 \mathrm{E}+06$ & f \\
\hline Os-191 & $2.20 \mathrm{E}+03$ & $4.96 \mathrm{E}-02$ & $4.44 E+04$ & $\mathrm{f}$ \\
\hline Os- 193 & $1.34 \mathrm{E}+04$ & $2.48 \mathrm{E}-02$ & $5.41 E+05$ & f \\
\hline Os-194 & $8.20 \mathrm{E}+01$ & $2.67 \mathrm{E}-01$ & $3.07 \mathrm{E}+02$ & $\mathrm{i}$ \\
\hline Ir-182 & $9.40 E+04$ & $1.36 \mathrm{E}-03$ & $6.89 E+07$ & $d$ \\
\hline Ir-184 & $3.20 \mathrm{E}+03$ & $5.67 \mathrm{E}-04$ & $5.64 \mathrm{E}+06$ & d \\
\hline Ir-185 & $2.60 \mathrm{E}+03$ & $2.15 \mathrm{E}-03$ & $1.21 E+06$ & d \\
\hline Ir -186 & $1.08 \mathrm{E}+03$ & $1.01 \mathrm{E}-03$ & $1.07 E+06$ & d \\
\hline
\end{tabular}


Table-Continued

\begin{tabular}{|c|c|c|c|c|}
\hline $\begin{array}{l}\text { ICRP-30 } \\
\text { Radionuclide }\end{array}$ & $\begin{array}{c}\text { Category } 3 \\
\text { Threshold } \\
\text { (Curies) }\end{array}$ & $\begin{array}{c}\text { Category } 3 \\
\text { Threshold (Grams) }\end{array}$ & $\begin{array}{l}\text { Calculated SA } \\
(\mathrm{Ci} / \mathrm{gm})^{5}\end{array}$ & Pathw \\
\hline Ir-187 & $7.40 \mathrm{E}+03$ & 4.63E-03 & $1.60 \mathrm{E}+06$ & d \\
\hline Ir-188 & $5.40 \mathrm{E}+02$ & $1.34 \mathrm{E}-03$ & $4.02 E+05$ & d \\
\hline Ir-189 & $1.26 \mathrm{E}+04$ & $2.43 \mathrm{E}-01$ & $5.19 E+04$ & $\mathbf{w}$ \\
\hline $\mathrm{Ir}-190 \mathrm{~m}$ & $2.00 \mathrm{E}+07$ & $1.45 \mathrm{E}+00$ & $1.38 \mathrm{E}+07$ & $\mathbf{i}$ \\
\hline Ir-190 & $6.80 \mathrm{E}+02$ & $1.20 \mathrm{E}-02$ & $5.68 E+04$ & d \\
\hline Ir-192m & $2.00 E+03$ & $2.59 \mathrm{E}+02$ & $7.73 E+00$ & $\mathrm{i}$ \\
\hline Ir-192 & $9.40 \mathrm{E}+02$ & $1.00 \mathrm{E}-01$ & $9.19 E+03$ & $d$ \\
\hline Ir-194m & $3.20 E+02$ & $8.13 \mathrm{E}-02$ & $3.94 E+03$ & $\mathrm{~d}$ \\
\hline Ir-194 & $1.42 E+04$ & $1.68 \mathrm{E}-02$ & $8.44 E+05$ & d \\
\hline Ir-195m & $1.10 \mathrm{E}+04$ & $2.60 \mathrm{E}-03$ & $4.23 E+06$ & $d$ \\
\hline Ir- 195 & $3.00 E+05$ & $4.67 \mathrm{E}-02$ & $6.43 E+06$ & $d$ \\
\hline Pt-186 & $8.00 E+03$ & $9.49 \mathrm{E}-04$ & $8.43 E+06$ & d \\
\hline Pt-188 & $2.60 \mathrm{E}+03$ & $3.82 \mathrm{E}-02$ & $6.81 E+04$ & f \\
\hline Pt-189 & $8.80 \mathrm{E}+03$ & $5.77 \mathrm{E}-03$ & $1.53 E+06$ & d \\
\hline Pt-191 & $4.40 \mathrm{E}+03$ & $1.81 \mathrm{E}-02$ & $2.43 E+05$ & d \\
\hline Pt-193m & $7.20 \mathrm{E}+03$ & 4.61E-02 & $1.56 \mathrm{E}+05$ & f \\
\hline Pt-193 & $2.40 \mathrm{E}+04$ & $6.48 \mathrm{E}+02$ & $3.71 E+01$ & f \\
\hline Pt- $195 \mathrm{~m}$ & $5.00 \mathrm{E}+03$ & $3.00 \mathrm{E}-02$ & $1.67 E+05$ & f \\
\hline Pt-197m & $1.98 E+05$ & $1.96 \mathrm{E}-02$ & $1.01 E+07$ & d \\
\hline Pt-197 & $3.20 \mathrm{E}+04$ & $3.68 \mathrm{E}-02$ & $8.70 E+05$ & f \\
\hline Pt-199 & $1.34 \mathrm{E}+05$ & 4.37E-03 & $3.07 E+07$ & $\mathrm{~d}$ \\
\hline Pt-200 & $1.58 \mathrm{E}+04$ & $1.26 \mathrm{E}-02$ & $1.25 \mathrm{E}+06$ & f \\
\hline Au-193 & $1.72 E+04$ & $1.87 \mathrm{E}-02$ & $9.20 \mathrm{E}+05$ & d \\
\hline $\mathrm{Au}-194$ & $1.16 \mathrm{E}+03$ & $2.84 \mathrm{E}-03$ & $4.09 E+05$ & $d$ \\
\hline Au-195 & $3.20 \mathrm{E}+03$ & $8.74 \mathrm{E}-01$ & $3.66 \mathrm{E}+03$ & f \\
\hline $\mathrm{Au}-198 \mathrm{~m}$ & $1.76 \mathrm{E}+03$ & $6.14 \mathrm{E}-03$ & $2.87 E+05$ & d \\
\hline $\mathrm{Au}-198$ & $2.00 \mathrm{E}+03$ & $8.20 \mathrm{E}-03$ & $2.45 E+05$ & d \\
\hline $\mathrm{Au}-199$ & $9.20 \mathrm{E}+03$ & $4.40 \mathrm{E}-02$ & $2.09 E+05$ & f \\
\hline $\mathrm{Au}-200 \mathrm{~m}$ & $5.60 \mathrm{E}+02$ & $6.68 \mathrm{E}-04$ & $8.38 E+05$ & d \\
\hline $\mathrm{Au}-200$ & $6.20 \mathrm{E}+04$ & $3.19 \mathrm{E}-03$ & $1.94 E+07$ & d \\
\hline $\mathrm{Au}-201$ & $8.40 \mathrm{E}+05$ & $2.37 \mathrm{E}-02$ & $3.54 E+07$ & d \\
\hline $\mathrm{Hg}-193 \mathrm{~m}$ & $1.08 \mathrm{E}+03$ & $7.38 \mathrm{E}-04$ & $1.46 \mathrm{E}+06$ & d \\
\hline $\mathrm{Hg}-193$ & $4.40 E+03$ & $9.48 \mathrm{E}-04$ & $4.64 E+06$ & $\mathrm{~d}$ \\
\hline $\mathrm{Hg}-194$ & $1.16 \mathrm{E}+01$ & $1.64 \mathrm{E}+00$ & $7.09 \mathrm{E}+00$ & f \\
\hline
\end{tabular}

Continued on next page 
Table-Continued

\begin{tabular}{|c|c|c|c|c|}
\hline $\begin{array}{c}\text { ICRP-30 } \\
\text { Radionuclide }\end{array}$ & $\begin{array}{c}\text { Category } 3 \\
\text { Threshold } \\
\text { (Curies) }\end{array}$ & $\begin{array}{c}\text { Category } 3 \\
\text { Threshold (Grams) }\end{array}$ & $\begin{array}{l}\text { Calculated SA } \\
(\mathrm{Ci} / \mathrm{gm})^{5}\end{array}$ & Pathu \\
\hline $\mathrm{Hg}-195 \mathrm{~m}$ & $5.00 \mathrm{E}+03$ & $1.29 \mathrm{E}-02$ & $3.86 \mathrm{E}+05$ & d \\
\hline Hg-195 & $1.28 \mathrm{E}+04$ & $7.89 \mathrm{E}-03$ & $1.62 \mathrm{E}+06$ & d \\
\hline $\mathrm{Hg}-197 \mathrm{~m}$ & $2.20 \mathrm{E}+04$ & $3.29 \mathrm{E}-02$ & $6.68 \mathrm{E}+05$ & f \\
\hline $\mathrm{Hg}-197$ & $2.00 \mathrm{E}+04$ & $8.06 \mathrm{E}-02$ & $2.48 \mathrm{E}+05$ & f \\
\hline $\mathrm{Hg}-199 \mathrm{~m}$ & $1.26 \mathrm{E}+05$ & $5.68 \mathrm{E}-03$ & $2.22 E+07$ & d \\
\hline $\mathrm{Hg}-203$ & $3.60 \mathrm{E}+02$ & $2.61 \mathrm{E}-02$ & $1.38 E+04$ & f \\
\hline $\mathrm{Tl}-194 \mathrm{~m}$ & $1.06 \mathrm{E}+04$ & $3.59 E-04$ & $2.96 \mathrm{E}+07$ & d \\
\hline T1-194 & $3.00 E+04$ & $1.02 \mathrm{E}-03$ & $2.94 \mathrm{E}+07$ & d \\
\hline T1-195 & $1.64 \mathrm{E}+04$ & $1.18 \mathrm{E}-03$ & $1.39 \mathrm{E}+07$ & d \\
\hline Tl-197 & $1.64 \mathrm{E}+04$ & $2.93 \mathrm{E}-03$ & $5.60 \mathrm{E}+06$ & d \\
\hline Tl-198m & $6.20 \mathrm{E}+03$ & 7.32E-04 & $8.46 E+06$ & d \\
\hline Tl-198 & $1.66 E+03$ & $5.56 \mathrm{E}-04$ & $2.99 E+06$ & d \\
\hline T1-199 & $1.14 \mathrm{E}+04$ & $5.37 \mathrm{E}-03$ & $2.12 E+06$ & d \\
\hline T1-200 & $9.20 \mathrm{E}+02$ & $1.53 \mathrm{E}-03$ & $6.01 E+05$ & $\mathrm{~d}$ \\
\hline Tl-201 & $4.20 \mathrm{E}+04$ & $1.97 \mathrm{E}-01$ & $2.13 E+05$ & d \\
\hline $\mathrm{T} 1-202$ & $1.84 \mathrm{E}+03$ & $3.48 \mathrm{E}-02$ & $5.29 E+04$ & d \\
\hline T1-204 & $1.20 \mathrm{E}+03$ & $2.59 \mathrm{E}+00$ & $4.64 E+02$ & f \\
\hline $\mathrm{Pb}-195 \mathrm{~m}$ & 4.40E+04 & $7.21 \mathrm{E}-04$ & $6.10 \mathrm{E}+07$ & d \\
\hline $\mathrm{Pb}-198$ & $1.46 \mathrm{E}+04$ & $2.21 \mathrm{E}-03$ & $6.60 \mathrm{E}+06$ & d \\
\hline Pb-199 & $1.06 \mathrm{E}+04$ & $1.01 \mathrm{E}-03$ & $1.05 \mathrm{E}+07$ & d \\
\hline $\mathrm{Pb}-200$ & $9.40 \mathrm{E}+03$ & $1.29 \mathrm{E}-02$ & $7.29 \mathrm{E}+05$ & d \\
\hline $\mathrm{Pb}-201$ & $2.80 \mathrm{E}+03$ & $1.69 \mathrm{E}-03$ & $1.66 \mathrm{E}+06$ & $\mathrm{~d}$ \\
\hline $\mathrm{Pb}-202 \mathrm{~m}$ & $1.74 E+03$ & $4.06 \mathrm{E}-04$ & $4.29 E+06$ & d \\
\hline $\mathrm{Pb}-202$ & $6.00 \mathrm{E}+01$ & $1.02 E+04$ & $5.90 \mathrm{E}-03$ & f \\
\hline $\mathrm{Pb}-203$ & $3.60 \mathrm{E}+03$ & $1.21 \mathrm{E}-02$ & $2.97 \mathrm{E}+05$ & d \\
\hline $\mathrm{Pb}-205$ & $2.40 \mathrm{E}+03$ & $1.97 \mathrm{E}+07$ & $1.22 \mathrm{E}-04$ & f \\
\hline $\mathrm{Pb}-209$ & $6.20 \mathrm{E}+05$ & $1.34 \mathrm{E}-01$ & $4.61 E+06$ & $\mathrm{i}$ \\
\hline $\mathrm{Pb}-210$ & $3.60 \mathrm{E}-01$ & $4.70 \mathrm{E}-03$ & $7.64 \mathrm{E}+01$ & f \\
\hline $\mathrm{Pb}-211$ & $6.20 \mathrm{E}+03$ & $2.51 \mathrm{E}-04$ & $2.47 E+07$ & $\mathrm{i}$ \\
\hline $\mathrm{Pb}-212$ & $3.20 \mathrm{E}+02$ & $2.30 \mathrm{E}-04$ & $1.39 \mathrm{E}+06$ & $\mathrm{i}$ \\
\hline $\mathrm{Pb}-214$ & $8.20 E+03$ & $2.50 \mathrm{E}-04$ & $3.28 E+07$ & $\mathrm{i}$ \\
\hline $\mathrm{Bi}-200$ & $9.40 \mathrm{E}+03$ & $3.64 \mathrm{E}-04$ & $2.58 \mathrm{E}+07$ & $\mathrm{~d}$ \\
\hline $\mathrm{Bi}-201$ & $9.40 \mathrm{E}+03$ & $1.09 \mathrm{E}-03$ & $8.66 E+06$ & d \\
\hline $\mathrm{Bi}-202$ & $2.00 \mathrm{E}+04$ & $2.15 \mathrm{E}-03$ & $9.29 E+06$ & d \\
\hline
\end{tabular}

Continued on next page 
Table-Continued

\begin{tabular}{|c|c|c|c|c|}
\hline $\begin{array}{l}\text { ICRP-30 } \\
\text { Radionuclide }\end{array}$ & $\begin{array}{c}\text { Category } 3 \\
\text { Threshold } \\
\text { (Curies) }\end{array}$ & $\begin{array}{c}\text { Category } 3 \\
\text { Threshold (Grams) }\end{array}$ & $\begin{array}{l}\text { Calculated SA } \\
(\mathrm{Ci} / \mathrm{gm})^{5}\end{array}$ & Pathway \\
\hline $\mathrm{Bi}-203$ & $8.40 \mathrm{E}+02$ & $6.40 \mathrm{E}-04$ & $1.31 E+06$ & d \\
\hline $\mathrm{Bi}-205$ & $8.80 \mathrm{E}+02$ & $2.11 \mathrm{E}-02$ & $4.16 \mathrm{E}+04$ & d \\
\hline $\mathrm{Bi}-206$ & $2.40 \mathrm{E}+02$ & $2.36 \mathrm{E}-03$ & $1.02 E+05$ & $\mathrm{~d}$ \\
\hline $\mathrm{Bi}-207^{(7)}$ & $5.00 \mathrm{E}+02$ & $1.10 \mathrm{E}+01$ & $4.55 \mathrm{E}+01$ & d \\
\hline $\mathrm{Bi}-210 \mathrm{~m}$ & $7.20 \mathrm{E}+00$ & $1.27 E+04$ & $5.68 \mathrm{E}-04$ & $\mathrm{i}$ \\
\hline $\mathrm{Bi}-210$ & $3.20 \mathrm{E}+02$ & $2.60 \mathrm{E}-03$ & $1.24 \mathrm{E}+05$ & $\mathrm{i}$ \\
\hline $\mathrm{Bi}-212$ & $2.00 \mathrm{E}+03$ & $1.37 \mathrm{E}-04$ & $1.46 \mathrm{E}+07$ & $\mathrm{i}$ \\
\hline $\mathrm{Bi}-213$ & $3.20 \mathrm{E}+03$ & $1.65 \mathrm{E}-04$ & $1.93 E+07$ & $\mathrm{i}$ \\
\hline $\mathrm{Bi}-214$ & $8.20 E+03$ & $1.86 \mathrm{E}-04$ & $4.42 E+07$ & $\mathrm{i}$ \\
\hline Po-203 & $1.76 \mathrm{E}+04$ & $6.97 \mathrm{E}-04$ & $2.52 E+07$ & d \\
\hline Po-205 & $5.40 \mathrm{E}+03$ & $6.36 \mathrm{E}-04$ & $8.49 E+06$ & d \\
\hline Po-207 & $1.88 \mathrm{E}+03$ & 7.24E-04 & $2.60 E+06$ & $d$ \\
\hline Po-210 & $1.90 \mathrm{E}+00$ & $4.20 \mathrm{E}-04$ & $4.49 \mathrm{E}+03$ & f \\
\hline At-207 & $7.40 \mathrm{E}+03$ & $8.80 \mathrm{E}-04$ & $8.41 E+06$ & d \\
\hline At-211 & $5.20 \mathrm{E}+03$ & $2.52 \mathrm{E}-03$ & $2.06 \mathrm{E}+06$ & $\mathrm{i}$ \\
\hline $\mathrm{Rn}-220$ & $2.00 \mathrm{E}+00$ & $2.17 \mathrm{E}-09$ & $9.22 E+08$ & $\mathrm{i}$ \\
\hline $\mathrm{Rn}-222$ & $1.00 \mathrm{E}+01$ & $6.50 \mathrm{E}-05$ & $1.54 \mathrm{E}+05$ & $\mathrm{i}$ \\
\hline Fr-222 & $5.20 \mathrm{E}+03$ & 8.84E-05 & $5.88 \mathrm{E}+07$ & $\mathrm{i}$ \\
\hline Fr-223 & $8.20 \mathrm{E}+03$ & 2.12E-04 & $3.87 E+07$ & $\mathrm{i}$ \\
\hline $\mathrm{Ra}-223$ & $6.20 \mathrm{E}+01$ & $1.20 \mathrm{E}-03$ & $5.12 E+04$ & f \\
\hline Ra-224 & $2.00 \mathrm{E}+02$ & $1.20 \mathrm{E}-03$ & $1.59 \mathrm{E}+05$ & $\mathrm{i}$ \\
\hline $\mathrm{Ra}-225$ & $7.20 \mathrm{E}+01$ & $1.80 \mathrm{E}-03$ & $3.92 E+04$ & $\mathrm{i}$ \\
\hline $\mathrm{Ra}-226$ & $1.20 \mathrm{E}+01$ & $1.21 \mathrm{E}+01$ & $9.89 \mathrm{E}-01$ & $\mathrm{f}$ \\
\hline $\mathrm{Ra}-227$ & $1.50 \mathrm{E}+05$ & $7.64 \mathrm{E}-03$ & $1.96 \mathrm{E}+07$ & $d$ \\
\hline $\mathrm{Ra}-228$ & $1.20 \mathrm{E}+01$ & $4.40 \mathrm{E}-02$ & $2.73 E+02$ & $\mathrm{f}$ \\
\hline Ac- 224 & $3.20 \mathrm{E}+03$ & $6.63 E-04$ & $4.83 E+06$ & i \\
\hline Ac- 225 & $3.20 \mathrm{E}+01$ & $5.50 \mathrm{E}-04$ & $5.80 \mathrm{E}+04$ & i \\
\hline Ac- 226 & $3.20 \mathrm{E}+02$ & $6.69 \mathrm{E}-04$ & $4.78 E+05$ & $\mathrm{i}$ \\
\hline Ac- 227 & $4.20 \mathrm{E}-02$ & $5.80 \mathrm{E}-04$ & $7.24 \mathrm{E}+01$ & $\mathrm{i}$ \\
\hline Ac- 228 & $9.40 \mathrm{E}+02$ & 4.19E-04 & $2.24 \mathrm{E}+06$ & $\mathrm{i}$ \\
\hline Th-226 & $1.04 \mathrm{E}+04$ & $3.86 \mathrm{E}-04$ & $2.69 E+07$ & $\mathrm{i}$ \\
\hline Th-227 & $3.20 \mathrm{E}+01$ & $1.04 \mathrm{E}-03$ & $3.07 \mathrm{E}+04$ & $\mathrm{i}$ \\
\hline Th-228 & $1.00 \mathrm{E}+00$ & $1.20 \mathrm{E}-03$ & $8.20 \mathrm{E}+02$ & $\mathrm{i}$ \\
\hline Th-229 & $9.40 \mathrm{E}-02$ & 4.42E-01 & $2.13 \mathrm{E}-01$ & i \\
\hline
\end{tabular}


Table-Continued

\begin{tabular}{|c|c|c|c|c|}
\hline $\begin{array}{l}\text { ICRP-30 } \\
\text { Radionuclide }\end{array}$ & $\begin{array}{c}\text { Category } 3 \\
\text { Threshold } \\
\text { (Curies) }\end{array}$ & $\begin{array}{c}\text { Category } 3 \\
\text { Threshold (Grams) }\end{array}$ & $\begin{array}{c}\text { Calculated SA } \\
(\mathrm{Ci} / \mathrm{gm})^{5}\end{array}$ & Pathway \\
\hline Th-230 & $6.20 \mathrm{E}-01$ & $3.10 \mathrm{E}+01$ & $2.02 \mathrm{E}-02$ & i \\
\hline Th-231 & $1.20 \mathrm{E}+04$ & $2.26 \mathrm{E}-02$ & $5.32 \mathrm{E}+05$ & d \\
\hline Th-232 & $1.00 \mathrm{E}-01$ & $9.10 \mathrm{E}+05$ & $1.09 \mathrm{E}-07$ & i \\
\hline Th-234 & $2.80 \mathrm{E}+03$ & $1.21 \mathrm{E}-01$ & $2.32 E+04$ & f \\
\hline $\mathrm{Pa}-227$ & $1.04 \mathrm{E}+04$ & 4.81E-04 & $2.16 \mathrm{E}+07$ & i \\
\hline $\mathrm{Pa}-228$ & $1.04 \mathrm{E}+03$ & $1.66 \mathrm{E}-03$ & $6.25 E+05$ & $\mathrm{i}$ \\
\hline $\mathrm{Pa}-230$ & $4.20 \mathrm{E}+02$ & $1.29 \mathrm{E}-02$ & $3.26 \mathrm{E}+04$ & $\mathrm{i}$ \\
\hline $\mathrm{Pa}-231$ & $2.00 \mathrm{E}-01$ & $4.23 \mathrm{E}+00$ & 4.73E-02 & $\mathrm{i}$ \\
\hline $\mathrm{Pa}-232$ & $1.02 \mathrm{E}+03$ & $2.37 \mathrm{E}-03$ & $4.30 E+05$ & d \\
\hline $\mathrm{Pa}-233$ & $4.60 \mathrm{E}+03$ & $2.22 \mathrm{E}-01$ & $2.08 E+04$ & d \\
\hline $\mathrm{Pa}-234$ & $1.52 \mathrm{E}+03$ & $7.60 \mathrm{E}-04$ & $2.00 \mathrm{E}+06$ & d \\
\hline U-230 & $3.20 \mathrm{E}+01$ & $1.17 \mathrm{E}-03$ & $2.73 \mathrm{E}+04$ & i \\
\hline U-231 & $9.80 \mathrm{E}+04$ & $7.28 \mathrm{E}-01$ & $1.35 \mathrm{E}+05$ & f \\
\hline $\mathrm{U}-232$ & $8.20 \mathrm{E}-01$ & $3.81 \mathrm{E}-02$ & $2.15 \mathrm{E}+01$ & $\mathrm{i}$ \\
\hline $\mathrm{U}-233$ & $4.20 \mathrm{E}+00$ & $4.40 \mathrm{E}+02$ & $9.68 \mathrm{E}-03$ & i \\
\hline $\mathrm{U}-234$ & $4.20 \mathrm{E}+00$ & $6.70 \mathrm{E}+02$ & $6.25 \mathrm{E}-03$ & $\mathrm{i}$ \\
\hline U-235 & $4.20 \mathrm{E}+00$ & $1.90 \mathrm{E}+06$ & $2.16 \mathrm{E}-06$ & $\mathrm{i}$ \\
\hline U-236 & $4.20 \mathrm{E}+00$ & $6.49 \mathrm{E}+04$ & $6.47 \mathrm{E}-05$ & $\mathrm{i}$ \\
\hline U-237 & $1.44 E+04$ & $1.76 \mathrm{E}-01$ & $8.16 E+04$ & d \\
\hline U-238 & $4.20 \mathrm{E}+00$ & $1.30 \mathrm{E}+07$ & $3.36 \mathrm{E}-07$ & $\mathbf{i}$ \\
\hline U-239 & $7.00 \mathrm{E}+06$ & $2.09 \mathrm{E}-01$ & $3.34 \mathrm{E}+07$ & d \\
\hline $\mathrm{U}-240$ & $1.40 \mathrm{E}+05$ & $1.51 \mathrm{E}-01$ & $9.26 \mathrm{E}+05$ & f \\
\hline Np-232 & $5.00 \mathrm{E}+04$ & $9.07 \mathrm{E}-04$ & $5.51 \mathrm{E}+07$ & d \\
\hline Np-233 & $3.00 \mathrm{E}+06$ & $1.35 \mathrm{E}-01$ & $2.23 E+07$ & d \\
\hline Np-234 & $7.80 \mathrm{E}+02$ & $6.15 \mathrm{E}-03$ & $1.27 \mathrm{E}+05$ & d \\
\hline Np-235 & $6.20 \mathrm{E}+04$ & $4.42 \mathrm{E}+01$ & $1.40 \mathrm{E}+03$ & f \\
\hline $\begin{array}{l}\mathrm{Np}-236 \\
(1.2 \mathrm{E}+5 \mathrm{yr})\end{array}$ & $2.40 \mathrm{E}+00$ & $1.90 \mathrm{E}+02$ & $1.26 \mathrm{E}-02$ & f \\
\hline $\begin{array}{l}\mathrm{Np}-236 \\
(22.5 \mathrm{hr})\end{array}$ & $4.20 \mathrm{E}+03$ & $7.11 \mathrm{E}-03$ & $5.90 \mathrm{E}+05$ & $\mathrm{i}$ \\
\hline Np-237 & $4.20 \mathrm{E}-01$ & $6.00 \mathrm{E}+02$ & $7.05 \mathrm{E}-04$ & $\mathrm{f}$ \\
\hline Np-238 & $1.30 \mathrm{E}+03$ & $5.00 \mathrm{E}-03$ & $2.59 \mathrm{E}+05$ & d \\
\hline Np-239 & $7.80 \mathrm{E}+03$ & $3.36 \mathrm{E}-02$ & $2.32 \mathrm{E}+05$ & d \\
\hline $\mathrm{Np}-240$ & $1.08 \mathrm{E}+04$ & 8.96E-04 & $1.21 \mathrm{E}+07$ & d \\
\hline
\end{tabular}

Continued on next page 
Table-Continued

\begin{tabular}{|c|c|c|c|c|}
\hline $\begin{array}{c}\text { ICRP-30 } \\
\text { Radionuclide }\end{array}$ & $\begin{array}{c}\text { Category } 3 \\
\text { Threshold } \\
\text { (Curies) }\end{array}$ & $\begin{array}{c}\text { Category } 3 \\
\text { Threshold (Grams) }\end{array}$ & $\begin{array}{l}\text { Calculated SA } \\
(\mathrm{Ci} / \mathrm{gm})^{5}\end{array}$ & Pathway \\
\hline Pu-234 & $2.00 \mathrm{E}+04$ & $1.31 \mathrm{E}-02$ & $1.52 E+06$ & $\mathrm{i}$ \\
\hline $\mathrm{Pu}-235$ & $3.20 \mathrm{E}+08$ & $1.01 \mathrm{E}+01$ & $3.16 E+07$ & i \\
\hline $\mathrm{Pu}-236$ & $2.00 \mathrm{E}+00$ & $3.76 \mathrm{E}-03$ & $5.32 \mathrm{E}+02$ & $\mathrm{i}$ \\
\hline $\mathrm{Pu}-237$ & $7.60 \mathrm{E}+04$ & $6.25 \mathrm{E}+00$ & $1.22 \mathrm{E}+04$ & f \\
\hline Pu-238 & $6.20 \mathrm{E}-01$ & $3.60 \mathrm{E}-02$ & $1.71 E+01$ & $\mathrm{i}$ \\
\hline $\mathrm{Pu}-239$ & $5.20 \mathrm{E}-01$ & $8.40 \mathrm{E}+00$ & $6.22 \mathrm{E}-02$ & $\mathrm{i}$ \\
\hline $\mathrm{Pu}-240$ & $5.20 \mathrm{E}-01$ & $2.28 \mathrm{E}+00$ & $2.28 \mathrm{E}-01$ & $\mathrm{i}$ \\
\hline $\mathrm{Pu}-241$ & $3.20 \mathrm{E}+01$ & $3.10 \mathrm{E}-01$ & $1.03 E+02$ & $\mathrm{i}$ \\
\hline $\mathrm{Pu}-242$ & $6.20 \mathrm{E}-01$ & $1.58 \mathrm{E}+02$ & $3.93 \mathrm{E}-03$ & i \\
\hline $\mathrm{Pu}-243$ & $1.14 E+05$ & $4.38 \mathrm{E}-02$ & $2.60 E+06$ & $\mathrm{~d}$ \\
\hline $\mathrm{Pu}-244$ & $6.20 \mathrm{E}-01$ & $3.49 E+04$ & $1.77 \mathrm{E}-05$ & $\mathrm{i}$ \\
\hline $\mathrm{Pu}-245$ & $6.20 E+03$ & $5.09 \mathrm{E}-03$ & $1.22 E+06$ & d \\
\hline Am-237 & $4.20 \mathrm{E}+04$ & $3.86 \mathrm{E}-03$ & $1.09 E+07$ & d \\
\hline Am-238 & $1.06 \mathrm{E}+04$ & $1.31 \mathrm{E}-03$ & $8.06 \mathrm{E}+06$ & d \\
\hline Am-239 & $1.60 \mathrm{E}+04$ & $1.45 E-02$ & $1.10 \mathrm{E}+06$ & d \\
\hline $\mathrm{Am}-240$ & $9.00 \mathrm{E}+02$ & $3.50 \mathrm{E}-03$ & $2.57 E+05$ & d \\
\hline Am-241 & $5.20 \mathrm{E}-01$ & $1.50 \mathrm{E}-01$ & $3.43 E+00$ & $\mathrm{i}$ \\
\hline $\mathrm{Am}-242 \mathrm{~m}$ & $5.20 \mathrm{E}-01$ & $5.30 \mathrm{E}-02$ & $9.72 E+00$ & $\mathrm{i}$ \\
\hline $\mathrm{Am}-242$ & $8.20 E+03$ & $1.01 \mathrm{E}-02$ & $8.09 E+05$ & $\mathrm{i}$ \\
\hline$A m-243$ & $5.20 \mathrm{E}-01$ & $2.60 \mathrm{E}+00$ & $1.99 \mathrm{E}-01$ & $\mathrm{i}$ \\
\hline Am-244m & $4.20 \mathrm{E}+05$ & $1.42 \mathrm{E}-02$ & $2.96 E+07$ & $\mathrm{i}$ \\
\hline Am-244 & $1.88 \mathrm{E}+03$ & $1.48 \mathrm{E}-03$ & $1.27 \mathrm{E}+06$ & d \\
\hline Am-245 & $3.40 \mathrm{E}+05$ & $5.45 \mathrm{E}-02$ & $6.24 E+06$ & $d$ \\
\hline Am-246m & $3.40 \mathrm{E}+04$ & $1.11 \mathrm{E}-03$ & $3.06 \mathrm{E}+07$ & $\mathrm{~d}$ \\
\hline$A m-246$ & $3.00 \mathrm{E}+04$ & $1.53 \mathrm{E}-03$ & $1.96 \mathrm{E}+07$ & $\mathrm{~d}$ \\
\hline $\mathrm{Cm}-238$ & $1.04 E+05$ & $1.89 \mathrm{E}-02$ & $5.49 E+06$ & $\mathrm{i}$ \\
\hline $\mathrm{Cm}-240$ & $5.20 \mathrm{E}+01$ & $2.58 \mathrm{E}-03$ & $2.02 E+04$ & $\mathrm{i}$ \\
\hline $\mathrm{Cm}-241$ & $1.90 \mathrm{E}+03$ & $1.15 \mathrm{E}-01$ & $1.65 E+04$ & d \\
\hline $\mathrm{Cm}-242$ & $3.20 \mathrm{E}+01$ & $9.70 \mathrm{E}-03$ & $3.31 \mathrm{E}+03$ & $\mathrm{i}$ \\
\hline $\mathrm{Cm}-243$ & $8.20 \mathrm{E}-01$ & $1.59 \mathrm{E}-02$ & $5.16 \mathrm{E}+01$ & i \\
\hline $\mathrm{Cm}-244$ & $1.04 \mathrm{E}+00$ & $1.28 E-02$ & $8.09 E+01$ & i \\
\hline $\mathrm{Cm}-245$ & $5.20 \mathrm{E}-01$ & $3.00 E+00$ & $1.72 \mathrm{E}-01$ & $\mathbf{i}$ \\
\hline $\mathrm{Cm}-246$ & $5.20 \mathrm{E}-01$ & $1.69 E+00$ & $3.07 E-01$ & $\mathbf{i}$ \\
\hline $\mathrm{Cm}-247$ & $6.20 \mathrm{E}-01$ & $6.68 E+03$ & $9.28 \mathrm{E}-05$ & $\mathrm{i}$ \\
\hline
\end{tabular}

Continued on next page 
Table-Continued

\begin{tabular}{ccccc}
\hline \hline $\begin{array}{c}\text { ICRP-30 } \\
\text { Radionuclide }\end{array}$ & $\begin{array}{c}\text { Category } 3 \\
\text { Threshold } \\
\text { (Curies) }\end{array}$ & $\begin{array}{c}\text { Category } 3 \\
\text { Threshold (Grams) }\end{array}$ & $\begin{array}{c}\text { Calculated SA } \\
(\mathrm{Ci} / \mathrm{gm})^{5}\end{array}$ & Pathway \\
\hline & & & & \\
Cm-248 & $1.04 \mathrm{E}-01$ & $2.44 \mathrm{E}+01$ & $4.25 \mathrm{E}-03$ & $\mathrm{i}$ \\
Cm-249 & $6.20 \mathrm{E}+05$ & $5.27 \mathrm{E}-02$ & $1.18 \mathrm{E}+07$ & $\mathrm{~d}$ \\
Bk-245 & $9.20 \mathrm{E}+03$ & $8.53 \mathrm{E}-02$ & $1.08 \mathrm{E}+05$ & $\mathrm{~d}$ \\
Bk-246 & $1.20 \mathrm{E}+03$ & $4.14 \mathrm{E}-03$ & $2.90 \mathrm{E}+05$ & $\mathrm{~d}$ \\
Bk-247 & $5.20 \mathrm{E}-01$ & $4.96 \mathrm{E}-01$ & $1.05 \mathrm{E}+00$ & $\mathrm{i}$ \\
Bk-249 & $2.00 \mathrm{E}+01$ & $1.22 \mathrm{E}-02$ & $1.64 \mathrm{E}+03$ & $\mathrm{i}$ \\
Bk-250 & $4.20 \mathrm{E}+03$ & $1.08 \mathrm{E}-03$ & $3.89 \mathrm{E}+06$ & $\mathrm{~d}, \mathrm{i}$ \\
Cf-244 & $6.20 \mathrm{E}+04$ & $1.56 \mathrm{E}-03$ & $3.97 \mathrm{E}+07$ & $\mathrm{i}$ \\
Cf-246 & $9.40 \mathrm{E}+02$ & $2.63 \mathrm{E}-03$ & $3.57 \mathrm{E}+05$ & $\mathrm{i}$ \\
Cf-248 & $9.40 \mathrm{E}+00$ & $5.95 \mathrm{E}-03$ & $1.58 \mathrm{E}+03$ & $\mathrm{i}$ \\
Cf-249 & $5.20 \mathrm{E}-01$ & $1.27 \mathrm{E}+00$ & $4.10 \mathrm{E}-01$ & $\mathrm{i}$ \\
Cf-250 & $1.04 \mathrm{E}+00$ & $9.51 \mathrm{E}-03$ & $1.09 \mathrm{E}+02$ & $\mathrm{i}$ \\
Cf-251 & $5.20 \mathrm{E}-01$ & $3.28 \mathrm{E}-01$ & $1.59 \mathrm{E}+00$ & $\mathrm{i}$ \\
Cf-252 & $3.20 \mathrm{E}+00$ & $5.95 \mathrm{E}-03$ & $5.38 \mathrm{E}+02$ & $\mathrm{i}$ \\
Cf-253 & $2.00 \mathrm{E}+02$ & $6.90 \mathrm{E}-03$ & $2.90 \mathrm{E}+04$ & $\mathrm{i}$ \\
Cf-254 & $2.00 \mathrm{E}+00$ & $2.35 \mathrm{E}-04$ & $8.50 \mathrm{E}+03$ & $\mathrm{i}$ \\
Es-250 & $1.64 \mathrm{E}+03$ & $2.75 \mathrm{E}-04$ & $5.97 \mathrm{E}+06$ & $\mathrm{~d}$ \\
Es-251 & $1.04 \mathrm{E}+05$ & $2.75 \mathrm{E}-01$ & $3.78 \mathrm{E}+05$ & $\mathrm{i}$ \\
Es-253 & $2.00 \mathrm{E}+02$ & $7.93 \mathrm{E}-03$ & $2.52 \mathrm{E}+04$ & $\mathrm{i}$ \\
Es-254m & $1.04 \mathrm{E}+02$ & $3.31 \mathrm{E}-04$ & $3.14 \mathrm{E}+05$ & $\mathrm{i}$ \\
Es-254 & $1.04 \mathrm{E}+01$ & $5.58 \mathrm{E}-03$ & $1.86 \mathrm{E}+03$ & $\mathrm{i}$ \\
Fm-252 & $1.04 \mathrm{E}+03$ & $1.90 \mathrm{E}-03$ & $5.48 \mathrm{E}+05$ & $\mathrm{i}$ \\
Fm-253 & $1.04 \mathrm{E}+03$ & $6.04 \mathrm{E}-03$ & $1.72 \mathrm{E}+05$ & $\mathrm{i}$ \\
Fm-254 & $1.04 \mathrm{E}+04$ & $2.73 \mathrm{E}-03$ & $3.81 \mathrm{E}+06$ & $\mathrm{i}$ \\
Fm-255 & $2.00 \mathrm{E}+03$ & $3.27 \mathrm{E}-03$ & $6.13 \mathrm{E}+05$ & $\mathrm{i}$ \\
Fm-257 & $2.00 \mathrm{E}+01$ & $3.96 \mathrm{E}-03$ & $5.06 \mathrm{E}+03$ & $\mathrm{i}$ \\
Md-257 & $1.04 \mathrm{E}+04$ & $4.43 \mathrm{E}-03$ & $2.35 \mathrm{E}+06$ & $\mathrm{i}$ \\
Md-258 & $3.20 \mathrm{E}+01$ & $3.48 \mathrm{E}-03$ & $9.21 \mathrm{E}+03$ & $\mathrm{i}$ \\
\hline \hline & & & & \\
\hline
\end{tabular}




\section{Notes, Explanations, and References}

1. DOE-STD-1027-92 "Hazard Categorization and Accident Analysis Techniques for Compliance with Doe Order 5480.23, Nuclear Safety Analysis Reports" (December 1992).

2. International Commision on Radiological Protection, Limits for Intakes of Radionuclides by Workers," ICRP Publication 30, Vols. I-IV, (Pergamon, Oxford 1980).

3. ICF Incorporated and C-E Environmental, EPA Contract 68-03-3452 "Technical Background Document to Support Final Rulemaking Pursuant to Section 102 of the Comprehensive Environmental Response, Compensation, and Liability Act: Radionuclides. (CERCLA 40 CFR 302.4) A report to the Emergency Response Division, Office of Emergency and Remedial Response, U.S. Environmental Protection Agency (February 1989).

4 The half-life of Bi-207 is $38 \mathrm{yr}$ according to ICRP-30 and another current source. However, the Radiological Health Handbook, U.S., (Department of Health Education And Welfare, Rockville, MD, 1970) and DOE/EH-0070, "External Dose-Rate Conversion Factors for Calculation of Dose to the Public," (July 1988) both give half-lives that are significantly lower (e.g., 28-33.4 yr). A 33.4-yr value agrees with DOE-STD-1027-92 Hazard Category 2 Threshold. ${ }^{7}$ In the calculations for Hazard Category 2, the 38-yr half-life leads to a Hazard Category 2 Threshold value about $12 \%$ larger than the DOE-STD-1027-92 value. The calculated Category 3 Threshold for Bi-207 given here in grams also reveals approximately a $12 \%$ difference with the DOE-STD-1027-92 value. DOE apparently is using a different SA in both places for this particular radionuclide.

Because ICRP-30 is the source for dose conversion factors, we think that, for consistency, the ICRP-30 half-live should be used for threshold calculations.

5. LANL calculated the SAs in Ci/gm directly from the half-life and atomic weight. The SA values in the table were calculated using the following formula:

$$
\begin{aligned}
& \mathrm{T}_{1 / 2}=\text { half-life }(\mathrm{yr}) \\
& \mathrm{AW}=\text { atomic weight of the isotope } \\
& \mathrm{N}_{\mathrm{o}}=6.023 \times 10^{23}=\text { Avagadro's Number } \\
& \quad \mathrm{SA}(\mathrm{Bq} / \mathrm{gm})=\left(0.693 \times \mathrm{N}_{\mathrm{o}}\right) /\left(\mathrm{AW} \times \mathrm{T}_{1 / 2} \times 3.154 \times 10^{7} \mathrm{sec} / \mathrm{yr}\right) \text { and } \\
& \mathrm{SA}(\mathrm{Ci} / \mathrm{gm})=\mathrm{SA}(\mathrm{Bq} / \mathrm{gm}) /\left(3.7 \times 10^{10} \mathrm{~Bq} / \mathrm{Ci}\right) .
\end{aligned}
$$

6. For the isotope H-3 (tritium), the threshold values are recommended to DOE by a special committee and are not calculated.

7. J. Clow, R. DeVore, J. Elder, G. Heindel, W. Inkret, and G. Miller, "Specific Activities and DOE-STD-1027-92 Hazard Category 2 Thresholds, LANL Fact Sheet," Los Alamos National Laboratory report, LA-12846-MS (November, 1994). 